Revue des patrimoines

\title{
Un grand homme dans le petit monde des grandes écoles
}

Les représentations de Pasteur dans les établissements d'enseignement supérieur parisien

\section{Christian Hottin}

\section{(2) OpenEdition}

Journals

Édition électronique

URL : http://journals.openedition.org/insitu/4410

DOI : $10.4000 /$ insitu.4410

ISSN : 1630-7305

Éditeur

Ministère de la culture

Référence électronique

Christian Hottin, « Un grand homme dans le petit monde des grandes écoles », In Situ [En ligne],

10 | 2009, mis en ligne le 19 mai 2009, consulté le 23 avril 2019. URL : http://journals.openedition.org/ insitu/4410 ; DOI : 10.4000/insitu.4410

Ce document a été généré automatiquement le 23 avril 2019.

\section{(c) (i) (9)}

In Situ Revues des patrimoines est mis à disposition selon les termes de la licence Creative Commons Attribution - Pas d'Utilisation Commerciale - Pas de Modification 4.0 International. 


\title{
Un grand homme dans le petit monde des grandes écoles
}

\author{
Les représentations de Pasteur dans les établissements d'enseignement
} supérieur parisien

\section{Christian Hottin}

\section{Avant-propos}

1 Au cours de l'année 2008, le Lahic a organisé, à l'initiative d'Odile Vincent et Noël Barbe, auxquels je me suis par la suite associé, un séminaire ayant pour thème "Pasteur en héros: l'institution d'un patrimoine scientifique? $»^{1}$. De nombreux aspects de la construction de la figure de Pasteur en héros y furent abordés. En premier lieu, on prêta une attention toute particulière aux différents lieux de vie de Pasteur transformés par la suite en lieux de sa mémoire, réceptacles de souvenirs et porteurs de discours plus ou moins aboutis sur sa figure: maisons de Dôle ou d'Arbois, domaine de Marnes-laCoquette, appartement de l'Institut Pasteur. Parallèlement, on porta son regard en direction des représentations de Pasteur dans l'espace public ou à destination du public : récits, films, monuments publics, images dans la littérature scolaire² ${ }^{2}$.

Quoique fortement inscrite dans la problématique de ce séminaire, la présente contribution offre une orientation quelque peu différente: en effet, il s'agit ici de s'attacher à des représentations de Pasteur élaborées dans un contexte particulier, celui $\mathrm{du}$ monde de l'enseignement supérieur parisien. Univers fait d'institutions dont l'ouverture au public peut être variable selon leur statut, mais qui sont aussi espaces de vie collégiale et communautaire pour leurs membres. Monde restreint, composé de quelques dizaines d'établissements qui tous, loin s'en faut, ne sont pas concernés par la problématique des représentations pastoriennes, mais monde renfermant de nombreuses singularités, des oppositions ou des contradictions qui peuvent parfois être révélées par l'analyse des métamorphoses d'un même objet au gré de ses pérégrinations institutionnelles, comme cela peut précisément être le cas avec Pasteur. 


\section{Introduction}

La question des représentations de Pasteur dans le monde de l'enseignement supérieur est l'occasion de revisiter, sous un angle d'approche inédit pour nous à ce jour, une recherche, menée dans le cadre d'une thèse de l'École nationale des chartes et dédiée à l'ensemble du patrimoine peint et sculpté des établissement d'enseignement supérieur parisiens (facultés universitaires, grandes écoles et grands établissements de recherche) ${ }^{3}$. Si jusqu'à présent l'étude de ce corpus d'œuvres à travers l'analyse des figurations multiples d'une personne unique n'a pas été envisagée, il faut s'en expliquer en reprenant brièvement le contexte intellectuel de ce travail, les hypothèses de recherche initiales et les problématiques ultérieurement envisagées. Pour rendre compte de ce patrimoine et le comprendre, trois courants de recherche, liés à trois disciplines différentes, ont été mobilisés, de manière plus ou moins explicite.

Depuis le courant des années 1970, à la suite des recherches pionnières de Maurice Agulhon ${ }^{4}$, l'anthropologie historique a profondément renouvelé l'histoire du XIXe siècle en intégrant à l'étude de celle-ci l'ensemble des artéfacts, uniques ou multiples, se rattachant à la vie politique de cette époque. À la suite des travaux portant sur l'émergence, les triomphes et les avatars de la figure de Marianne ${ }^{5}$, bustes et statues ${ }^{6}$, décors de mairies ${ }^{7}$ ou d'administrations, sont devenus pour les historiens objets d'étude à part entière (avec l'active collaboration des conservateurs et des historiens d'art) et non plus seulement matériaux pédagogiques.

C'est encore au cours de cette décennie qu'une part importante de la production artistique du XIXe siècle, jusqu'alors largement dépréciée, fait l'objet d'une réévaluation complète. Ces années débutent par la destruction des halles de Baltard, elles se terminent avec la promesse du futur musée d'Orsay. Des architectes, et notamment Viollet-le-Duc ${ }^{8}$, sont redécouverts, des œuvres sont sauvées ${ }^{9}$, une nouvelles génération d'historiens d'art réhabilite l'art issu de l'enseignement de l'École des beaux-arts ${ }^{10}$. Pour qui souhaitait aborder le patrimoine des établissements d'enseignement supérieur en croisant les approches propres à l'histoire et à l'histoire de l'art, ces deux ensembles de travaux s'imposaient comme autant de guides et de grilles de lecture.

6 Plus indirecte et moins aisée était la mobilisation de recherches issues des sciences sociales, et notamment les travaux de Pierre Bourdieu portant sur le monde universitaire ${ }^{11}$ ou sur celui des grandes écoles. Celle-ci s'avérait pourtant nécessaire et particulièrement profitable du moment que les œuvres cessaient d'être envisagées uniquement dans leur relation matérielle avec l'architecture qui les abritait ou avec l'institution qui habitait cette architecture, en d'autres termes dès qu'était posée la question de la relation entre les groupes et individus peuplant des bâtiments et constituant ces institutions, et les peintures ou sculptures par eux créées ou suscitées.

Il s'agissait dès lors de tenter de comprendre quel rôle les œuvres avaient pu jouer dans la vie des groupes (élèves, anciens élèves, professeurs ou administrateurs issus de l'institution) : quels avaient été les processus de décision conduisant à leur réalisation (et éventuellement influant sur le traitement du sujet), quelles avaient été les formes et les modalités de leur réception par ces mêmes groupes, puis, dans la durée, de tenter de saisir les éventuelles appropriations dont elles auraient pu faire l'objet. L'objectif étant en définitive de savoir si ces peintures et sculptures, bien loin d'en être un donné d'emblée, 
avaient pu devenir un patrimoine pour ces institutions, et contribuer à créer et à entretenir un sentiment de communauté parmi leurs membres.

À côté des phénomènes d'identification existant entre les membres d'un groupe et les œuvres s'y rapportant, il s'agissait de considérer également les interactions entre les groupes (phénomènes d'émulation, de rivalité, de vassalité) et la manière dont celles-ci pouvaient éventuellement recevoir une traduction iconique sous la forme d'une œuvre d'art.

Trois axes de recherche devaient en définitive être envisagés. En premier lieu, la capacité pour un groupe d'affirmer son identité à travers la production d'œuvres (avec ou sans médiatisation par le biais de la commande publique). En deuxième lieu, l'affirmation de cette identité par la caractérisation de ses traits constitutifs (ici prend place notamment l'invocation récurrente de la figure des fondateurs ou des membres illustres de l'établissement). En troisième lieu enfin, la volonté souvent affichée de se différencier des autres groupes par l'affirmation de sa supériorité (qui peut revêtir des formes variées: ancienneté, notoriété).

10 L'étude des représentations pastoriennes dans l'espace universitaire parisien, tout en livrant une nouvelle facette de la construction de cette figure héroïque, est l'occasion de retrouver, d'établissement en établissement, quelques-uns de ces axes d'étude d'une recherche antérieure.

\section{Le contexte historique et artistique : la double singularité de Pasteur}

\section{La société des héros}

11 La société française du XIXe siècle demeure marquée par la quête du héros. Ainsi que l'a fait remarquer Daniel Fabre dans son introduction au présent séminaire ${ }^{12}$, une des conséquences majeures de la Révolution française et du régicide réside dans la perte de ce référent à la fois symbolique et physique que constituait la personne royale. Cette rupture dans l'unité du corps politique de la Nation perdure tout au long du siècle, en même temps que se manifestent, principalement par le biais des allégories ou de la multiplication des figures héroïques, les recherches de restauration de ces symboles d'unité retrouvée.

12 Ce qui est vrai pour le corps social dans son ensemble peut également, selon nous, s'appliquer aux différentes institutions particulières qui le structurent. La rupture politique de la Révolution est également une rupture institutionnelle profonde, une refondation de la plupart des corps constitués, des administrations et des établissements publics. Les institutions du XIXe siècle, telles que le Conseil d'État, la Cour des comptes, les cours de justice, les grands départements ministériels, les facultés ou nombre d'établissements d'enseignement supérieur sont soit des institutions neuves, issues de la Révolution et de l'Empire, soit des institutions existant sous l'Ancien Régime, mais profondément renouvelées et pour ainsi dire refondées par les régimes issus de 1789 . Dans un pays qui connaît une instabilité politique chronique, elles sont souvent des facteurs de stabilité et de continuité, mais connaissent elles aussi ce besoin d'asseoir leur légitimité. Dans les discours, les textes, l'architecture ou les représentations iconiques, elles y parviennent soit en invoquant la continuité avec leurs devancières disparues, soit 
en mettant en avant des figures abstraites ou héroïques qui contribuent à conforter leur identité nouvelle en cours de construction.

Pour nombre d'entre elles (que l'on songe par exemple aux «écoles de l'An III»: Polytechnique, le Cnam, l'École des langues orientales, l'École normale supérieure), la décennie 1890-1900 correspond au premier centenaire de leur existence et est marquée par une poussée de fièvre commémorative tout à fait comparable mutatis mutandis à celle qu'a connue la France après 1989. Déjà nombreux auparavant, les héros de ces établissements, morts ou vifs, se multiplient au gré des éloges écrits ou picturaux, sculptés ou musicaux.

14 Avant d'aborder le cas de Pasteur, il faut préciser brièvement les conditions techniques et administratives de la multiplication de ces images.

La reproductibilité des images par le biais de nouveaux procédés de gravure ou par la photographie en est une des conditions techniques les plus évidentes. Les conséquences s'en manifestent dans la presse et dans l'édition. On ne reviendra pas sur le cas des manuels scolaires, renvoyant pour cela aux analyse désormais classiques de Christian $A m a l v i^{13}$ ou aux travaux plus récents, par exemple ceux de Laurent Avezou ${ }^{14}$.

16 La question de la figuration des héros - ou des allégories - dans l'espace public obéit à des règles particulières, à mettre en relation avec les principes de l'architecture (et la place que les peintures ou les sculptures doivent avoir dans la physionomie et l'économie générale d'un édifice, notamment public) ou avec ceux de l'urbanisme. Dans le cas des monuments peints ou sculptés (mais aussi épigraphiques) destinés à un bâtiment officiel, ceux-ci viennent renforcer le caractère parlant de ces architectures, pour lesquelles les élévations, le choix du vocabulaire architectural et décoratif (entre autres l'ordre des colonnes et des chapiteaux) doivent autant que possible se conformer aux qualités et à l'identité de l'institution ainsi incarnée dans ces formes (légèreté et gaîté pour un théâtre, sobriété et gravité pour un tribunal, sévérité et rudesse pour une prison).

17 Enfin, cette profusion de décors est concrètement rendue possible par un système de commande publique associant institutions, administration des Beaux-Arts, architectes des Bâtiments civils et artistes. Les premières, en général avec l'accord des troisièmes, établissent la liste de leurs desiderata en matière de décoration et l'adressent à la deuxième, laquelle assure, en concertation avec l'architecte (et souvent en fonction du réseau de relations de ce dernier), la mise en correspondance entre une demande de représentation et un artiste qui sera en charge de sa réalisation. Comme on a pu le constater à loisir, le résultat est souvent assez décevant, ces travaux très répétitifs (en particulier dans le cas des bustes les plus ordinaires, invariablement payés 2400 francs par l'administration) n'étant pour des sculpteurs souvent nécessiteux que des commandes « alimentaires » aboutissant à des œuvres banales, riches en stéréotypes plus qu'en qualités expressives ou stylistiques ${ }^{15}$. Cette faiblesse était connue et reconnue dès le temps de la création de ces œuvres et périodiquement déplorée par les inspecteurs des Beaux-Arts. Moins sérielles, les commandes de peintures ${ }^{16}$ présentaient un caractère plus exceptionnel : les commandes de portraits étant relativement rares, les toiles (le plus souvent marouflées) étaient destinées à des espaces de représentation (amphithéâtre, salle de conseil) et traitaient de sujets dûment choisis et parfois longuement discutés. 


\section{Pasteur, héros singulier}

18 Si la plupart des individus portraiturés ou sculptés dans les institutions peuvent être considérés avant tout comme des héros "locaux", peu connus en dehors de leur établissement, il n'en va pas de même pour Louis Pasteur dont la valeur hérö̈que s'affirme globalement, dans l'ensemble des espaces particuliers de la société tout autant que dans l'espace commun et public de la ville capitale.

Figure 1

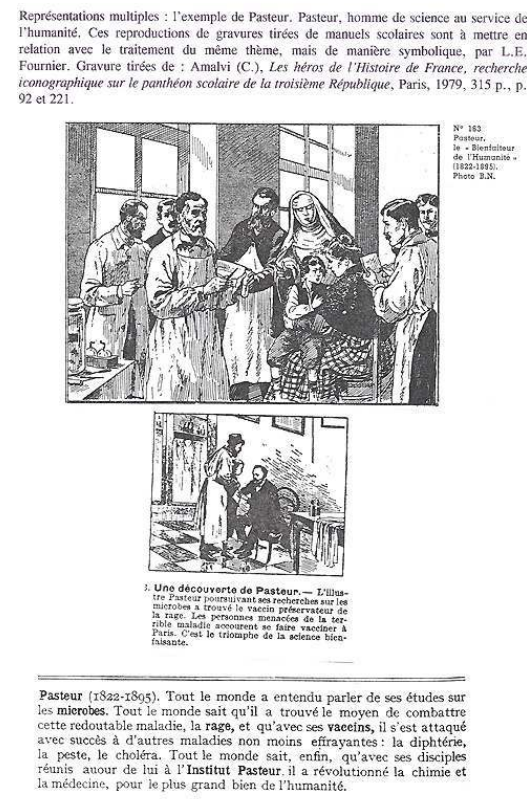

Pasteur dans les manuels scolaires de la Troisième République. Phot. C. Hottin.

(c) Coll. part.

19 Ainsi que l'a montré Christian Amalvi, Pasteur est un sujet de choix pour les manuels scolaires de la Troisième République. (fig. $\mathbf{n}^{\circ}$ 1) De même, dans l'espace parisien, sa présence est fortement marquée par la toponymie officielle (avec un boulevard et une station de métropolitain), et par les dérivés privés de celle-ci (on songe ici aux raisons sociales de nombreux commerces ou débits de boisson situés à proximité de la station ou sur le tracé du boulevard). (fig. $\mathbf{n}^{\circ} 2$ ) 
Figure 2

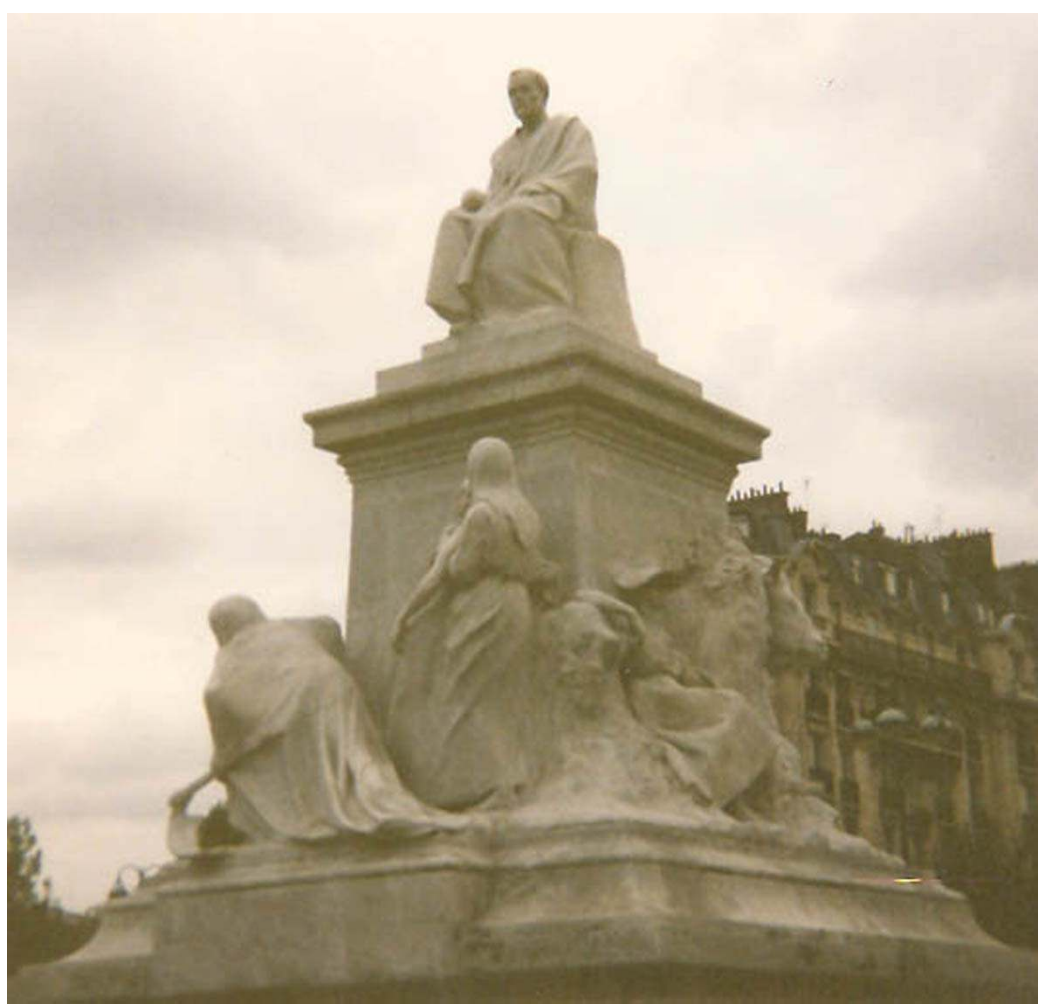

Le monument à Pasteur de l'avenue de Breteuil, par Falguière. Phot. C. Hottin.

(c) Coll. part.

Mais la présence de Pasteur dans l'espace public est avant signifiée par la monumentale sculpture de l'avenue de Breteuil, monument de marbre à figures multiples érigé grâce à une souscription internationale. On verra du reste que cette œuvre majeure entretient plus d'une relation avec de plus modestes monuments conservés dans les établissements d'enseignement supérieur.

21 Face à cette abondante diffusion de la figure et du nom du héros dans la littérature pédagogique et dans l'espace urbain, les figurations de Pasteur dans les facultés et les grandes écoles peuvent apparaître comme autant de déclinaisons locales et localisées de la figure d'un héros national ou global. Les étudier suppose donc de revenir sur le parcours académique de Louis Pasteur et sur les relations qu'il a entretenues avec ses divers établissements.

Né en 1822, Pasteur a brièvement fréquenté l'institution Barbet en 1838 avant de revenir à Besançon. Bachelier ès lettres en 1840, il est reçu bachelier ès sciences en 1842 et est admis à l'École normale l'année suivante. Il soutient sa thèse en 1847. Il enseigne ensuite aux facultés des Dijon et Strasbourg, puis dans celle de Lille, où il assure la fonction de doyen. Cette expérience mêlant activités scientifiques et administratives paraît lui convenir, puisqu'il revient à l'École normale en 1857 en qualité d'administrateur chargé de la direction des études. Se placent alors ces premiers grands travaux de chercheur, notamment la réfutation de la génération spontanée. En 1867, jugé trop sévère par ses collègues et les élèves de l'école, il abandonne ses fonctions d'administrateur et démissionne. Il obtient alors une chaire en Sorbonne, en même temps que la création d'un laboratoire de chimie physiologique à l'École. Tel sera le cadre de son travail 
jusqu'en 1888, date de son installation à l'Institut Pasteur. Outre ses fonctions principales, Pasteur en a assumé de nombreuses autres, dont celle peu connue de chargé d'enseignement de la chimie à l'École des beaux-arts ${ }^{17}$.

L'univers scientifique dans lequel évolue Pasteur est marqué par une double césure, institutionnelle et chronologique. Institutionnelle, car cet univers porte la marque de la dichotomie caractéristique du système français entre grandes écoles (ici principalement l'École normale), qui sont le lieu de formation des élites, et les espaces de production du savoir scientifique et les facultés, qui sont surtout le lieu de diffusion de ce savoir, associé au monopole de la collation des grades. Chronologique, car son parcours de savant passe par la défaite de 1871, qui a profondément contribué à transformer le paysage matériel, intellectuel et institutionnel de la science française. Chercheur au sein de l'École normale, Pasteur œuvre dans l'un des rares lieux de recherche organisé du paysage savant parisien. Dès le milieu des années 1860 , à travers plusieurs publications, il tente en vain d'alerter les pouvoirs publics sur les faiblesses du système académique national ${ }^{18}$, particulièrement patentes quand on le compare à l'organisation des universités allemandes. Le monde qu'il connait, et contribue à façonner, après 1871 est celui de la recréation des universités en France, du développement des séminaires et des laboratoires, de la reconstruction partielle ou totale des grands établissements scientifiques (École de pharmacie, Sorbonne ${ }^{19}$, Collège de France, faculté de médecine, etc.).

\section{Variations sur la présence de Pasteur dans le monde de l'enseignement supérieur parisien}

\section{Représentations multiples en un lieu unique - Représentations uniques en des lieux multiples}

Pour s'en tenir à une stricte comptabilité, il apparaît que les représentations de Pasteur sont très inégalement réparties entre les différents établissements d'enseignement supérieur parisiens. En effet, on observe d'une part la présence de plusieurs images de lui au sein d'un même établissement, et d'autre part des figurations uniques, disséminées dans diverses écoles qui ont toutes en commun d'être vouées à l'enseignement des sciences de la vie.

L'École normale supérieure est l'institution qui, avec six représentations, se détache le plus nettement. On aura l'occasion de revenir sur les raisons de cette profusion, qui est du reste double: si aucune école ne rassemble autant de représentations de notre héros, il est aussi le héros le plus honoré au sein de cet établissement ${ }^{20}$. La Sorbonne mérite également une attention particulière, avec deux représentations.

À contrario, beaucoup d'édifices accueillent une seule figuration du savant. L'Institut de chimie (œuvre tardive de Nénot, devenu aujourd'hui École nationale supérieure de chimie de Paris) mérite d'être cité, bien que Pasteur apparaisse de manière assez indistincte dans une vaste composition de Georges Leroux conservée dans l'actuelle bibliothèque. L'École nationale vétérinaire de Maisons-Alfort possède une petite tête, œuvre du sculpteur Dubois, dont on trouve un autre exemplaire à l'École normale. Un buste est visible dans la bibliothèque de l'Institut national agronomique, fort peu mis en valeur au demeurant. Ces deux représentations sont des œuvres isolées. Il n'en va pas de même pour l'imposante 
statue installée dans une des cages d'escalier de la faculté de pharmacie de l'avenue de l'Observatoire (actuelle université Paris V): le chimiste y figure en compagnie de Marcelin Berthelot, Louis Arago et Claude Bernard.

\section{Du portrait psychologique au héros éternel : quels visages de Pasteur?}

\section{Un exemple : La tête de Pasteur par Dubois à l'École normale supérieure}

Figure 3

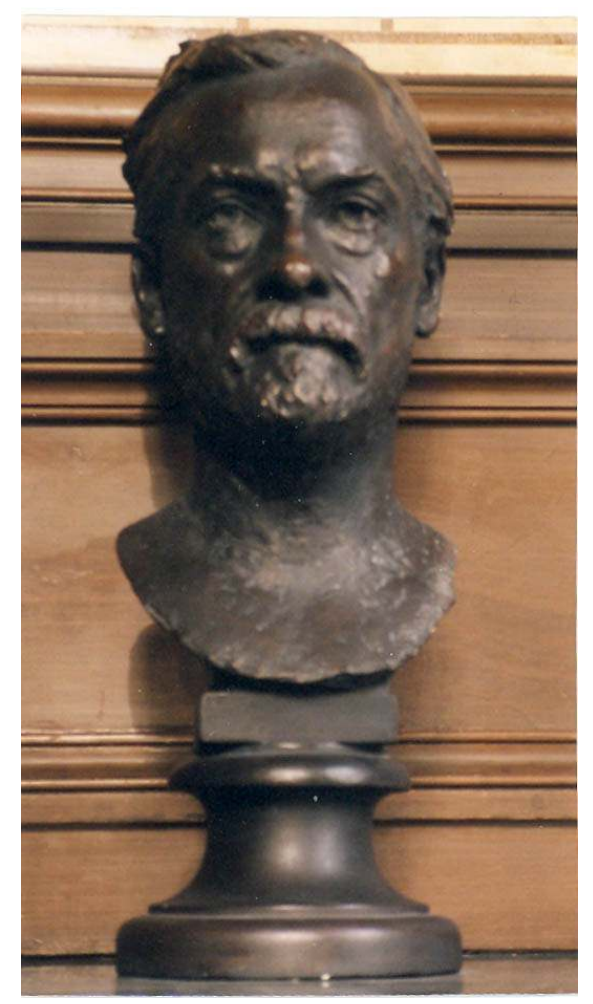

Un exemple : la tête de Pasteur par Dubois à l'École normale supérieure. Phot. C. Hottin.

(c) Coll. part.

27 (fig. $\mathbf{n}^{\circ}$ 3) Cette œuvre de dimensions réduites semble avoir été achetée à la demande de Paul Dupuy pour compléter le monument érigé dans le jardin de l'École normale. Elle était destinée à la salle des actes où elle devait retrouver le buste de Fustel de Coulanges et les noms des autres normaliens célèbres inscrits sur les plaques de marbre. L'intention de Dupuy et de Lavisse était de rendre ainsi plus présent le souvenir de l'illustre savant :

«L'image de pasteur sera ainsi présente à nos cérémonies, à nos réunions amicales, et son souvenir sera plus vivant pour ceux de nos jeunes camarades qui n'ont pu le connaître $»^{21}$. Le buste ne se trouve plus aujourd'hui dans la salle des actes, il a pris place dans le bureau de Pasteur transformé en lieu de mémoire.

30 Cette tête de Pasteur peut apparaître comme l'antithèse de tableau célèbre peint par Edelfeldt et conservé au musée d'Orsay, si souvent reproduit, aussi bien que du monument de l'avenue de Breteuil. Aucune mise en scène pour évoquer ici le difficile labeur de la recherche scientifique, pas de figures annexes à valeur symbolique ou 
allégorique pour exprimer la gratitude de la société envers le bienfaiteur de l'humanité. La sculpture se résume à la tête, fait l'économie du torse, habituel prétexte à l'évocation de la dignité sociale et de la position éminente du sujet représenté : la tête, dépouillée de tout élément accessoire, s'impose comme un portrait psychologique par excellence. Rien ne vient détourner de l'intensité du regard, le visiteur est frappé par la mise volontaire, impérieuse, autoritaire, du visage.

Cette représentation de Pasteur mérite d'autant plus qu'on s'y attarde que de tels portraits essentiellement psychologiques sont peu fréquents dans les représentations d'universitaires de la fin du XIXe siècle. Si les sculpteurs s'efforcent de rendre les traits avec fidélité, le rendu du costume, et notamment de la toge, tient également une grande place dans le travail de l'artiste. La figuration de cet élément du costume, qui qualifie le personnage en tant que savant universitaire, est d'autant plus importante que sur la toge sont épinglées les décorations reçues par celui qui la porte. Ainsi elle se transforme en curriculum vitae visuel. Selon un procédé identique, militaires, ingénieurs de grands corps, membres de l'Institut de France sont à cette époque presque systématiquement représentés dans l'uniforme de leur compagnie. On peut opposer à ce portrait de Pasteur réduit à l'essentiel le buste en plâtre de la bibliothèque de l'Institut agronomique : il y est représenté en bourgeois de son temps, sanglé dans une veste étroitement ajustée, l'allure stricte, la mine banalement sévère. (fig. $\mathbf{n}^{\circ}$ 4)

Figure 4

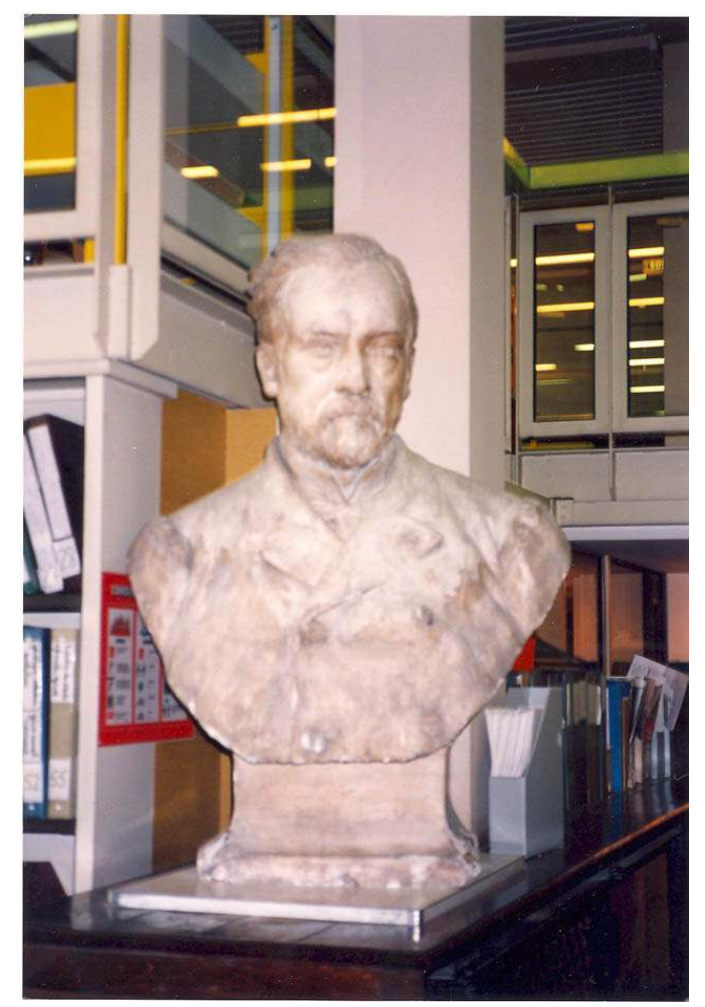

Le buste de Pasteur à I'Institut national Agronomique. Phot. C. Hottin.

(c) Coll. part.

Autre exemple : Pasteur en « grand absent » l'œuvre de Leroux à l'Institut de chimie 


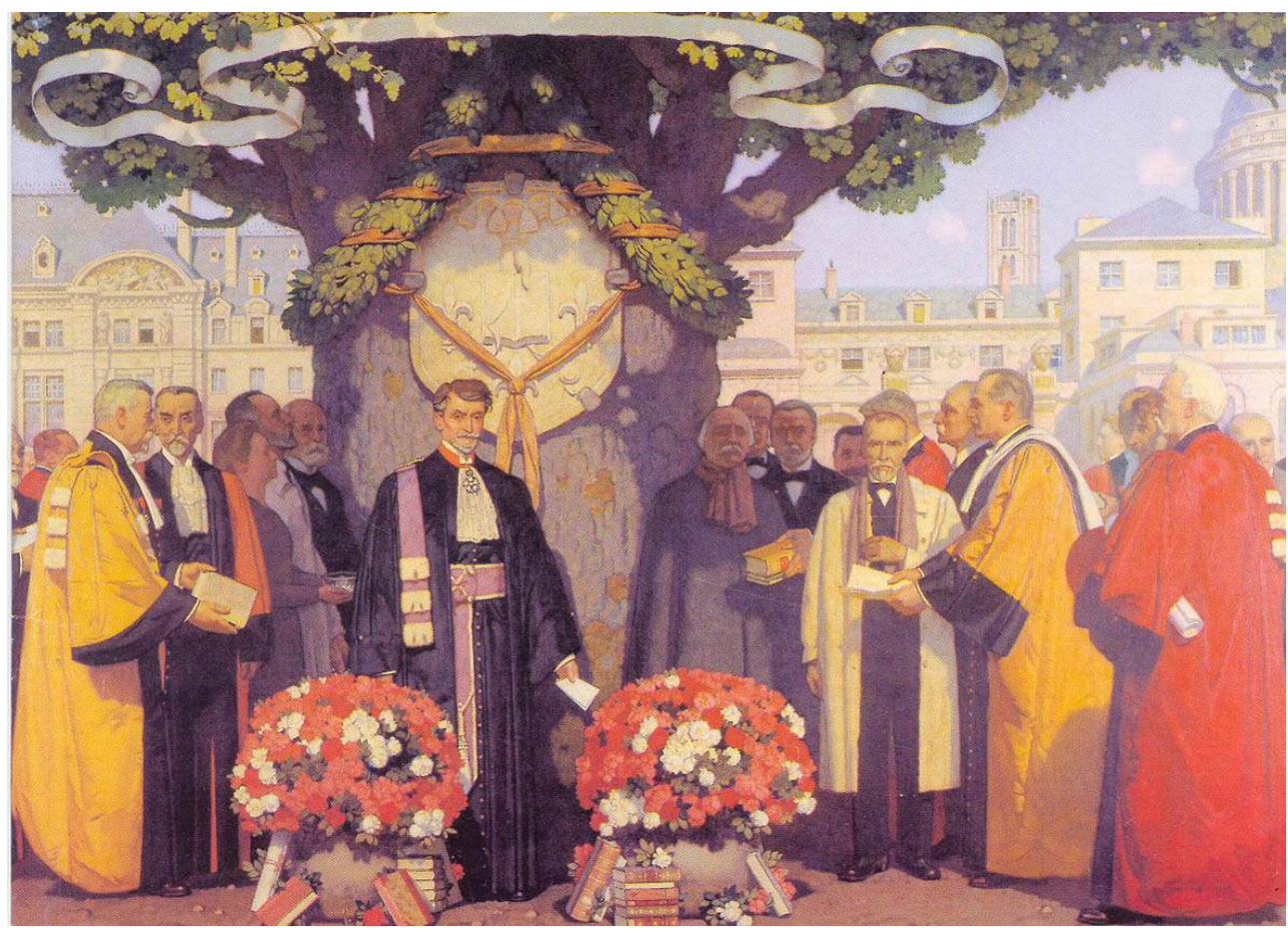

Pasteur « en grand absent », l'œuvre de Leroux à I'Institut de chimie. Phot. J.C. Doerr.

(fig. $\mathbf{n}^{\circ}$ 5) Si Pasteur ne figure pas au centre de cette composition, elle n'en mérite pas moins d'être évoquée tant en raison de l'originalité de son sujet que du traitement qui en est fait. Gaston Leroux a peint une fête imaginaire : les lieux sont fictifs, des morts et des vivants s'y côtoient, un chêne de légende étend sont ombre sur toute l'assemblée. En concevant son œuvre de cette manière, il donne à voir une célébration de la gloire de l'université de Paris d'une puissance inégalée. À l'arrière-plan nous reconnaissons, présentés les uns à côté des autres, les principaux monuments du Paris universitaire, reconstruits ou agrandis par la République: l'observatoire, la Sorbonne de Nénot, le Collège de France, la tour Clovis du lycée Henri IV, le Panthéon, la maison internationale de la Cité internationale universitaire. Une fête se tient devant ce grandiose panorama. On distingue la foule massée sur les gradins (panneaux latéraux).

$\mathrm{Au}$ premier plan sont représentées les personnalités les plus éminentes du monde universitaire de l'entre-deux-guerres. Le dessin de Leroux est net, d'une précision presque photographique, et chaque visage est de ce fait aisément identifiable. Les doyens entourent le recteur Sébastien Charléty. La liste des personnalités présente est édifiante : René et Paul Hazard, Maurain, Chabrier, Carcopino, Chabrier, Capitant, Urbain, Nénot, Barthélémy, Perrin, Vaillant, Marie, Bédier, Chauvette, Marquis, Roux, etc.

Le recteur se tient debout sous un chêne énorme, vivant symbole de l'université. Cet arbre porte sur son tronc les armes de l'institution. Telle une divinité protectrice, il couvre tous les présents, morts ou vifs. En effet, sous l'ombre de son feuillage, les traits comme estompés mais tous parfaitement reconnaissable, sont massés les grands héros de la science du tournant du siècle, auxquels Leroux a tenu à faire une place : on y trouve, bien entendu, Louis Pasteur, en compagnie, encore une fois, de Claude Bernard et de Marcelin Berthelot. Pierre et Marie Curie se sont joints à eux. Par-delà la mort, mais pour 
ainsi dire immortel dans la science, Pasteur se joint donc aux savants présents et passés pour célébrer la grandeur de l'université de Paris.

À contrario, la statue de l'escalier de l'École de pharmacie, qui le représente "à l'antique ", vêtu d'une toge, et donc hors contexte historique ou institutionnel précis, serait à rapprocher de la statue de l'avenue de Breteuil, bel exemple d'un Pasteur éternel, quasiment divinisé, plus qu'immortel.

\section{De la confrontation des images à l'esquisse d'un portrait}

En définitive, Ces multiples représentations de Pasteur dessinent l'image d'une figure complexe, qui dépasse les clivages institutionnels. On le rencontre, comme de juste, à l'École normale et à la Sorbonne, mais aussi dans divers établissements avec lesquels il n'entretint pas de relations de grande proximité.

Personnage ancré dans une époque, il peut être figuré dans un contexte historique précis, daté, mais également figurer comme symbole d'une science (la chimie), voire comme symbole des sciences dans leur ensemble (face aux lettres, incarnées à la Sorbonne par Victor Hugo) ou de la science dans sa globalité.

Cette approche générale des représentations de Pasteur doit maintenant être approfondie par l'étude circonstanciée d'un certain nombre de ces figurations, mises en relation avec leur contexte spatial et institutionnel précis

\section{L'École normale : la cinquième demeure de Pasteur?}


Figure 6

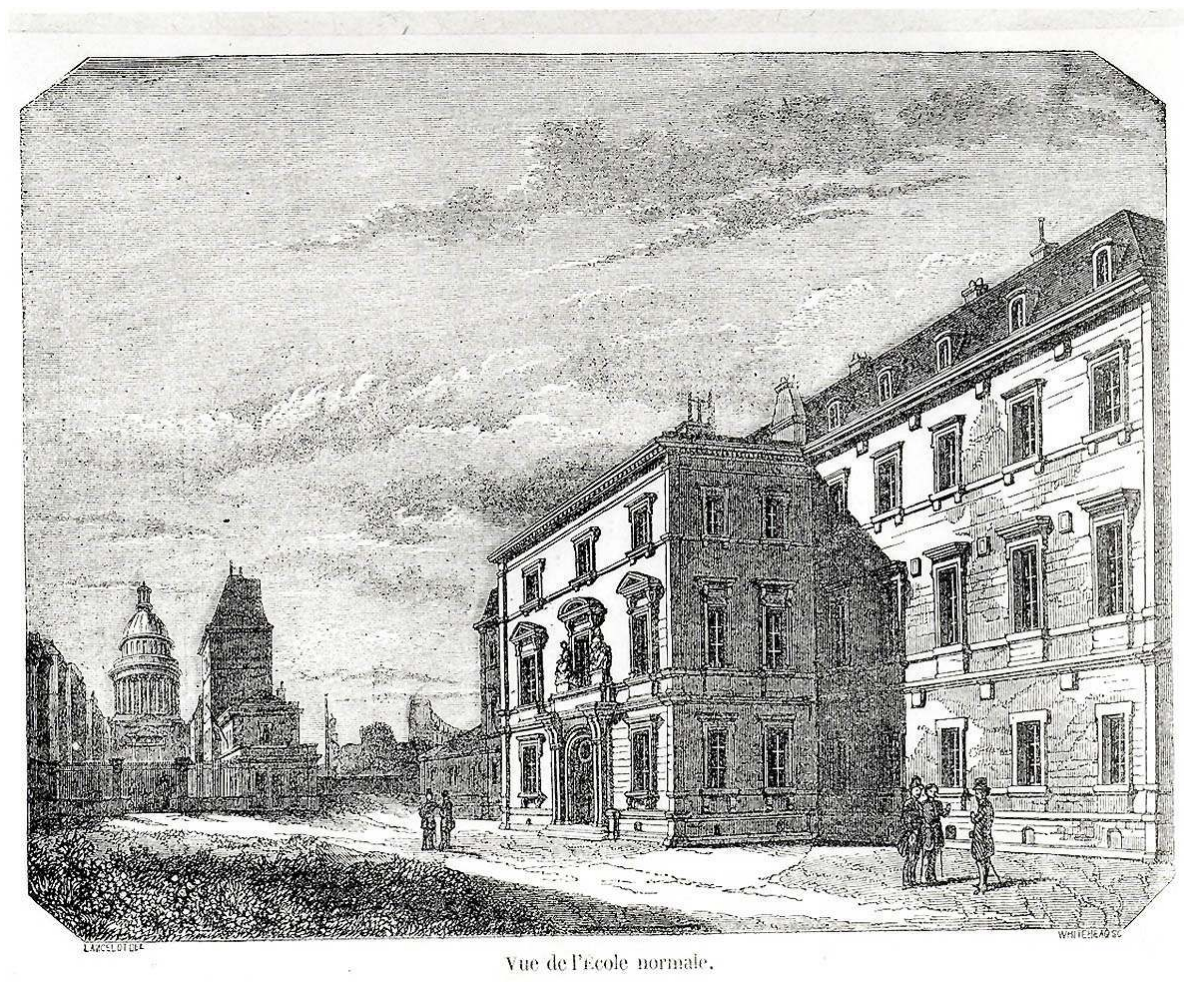

La rue d'Ulm et l'École normale dans les années 1850. Phot. C. Hottin.

(c) Coll. part.

Après Arbois, Dôle, Marnes-la-Coquette et l'institut qui porte son nom, l'école de la rue d'Ulm peut à juste titre apparaître comme un autre lieu pastorien de grande importance. (fig. $n^{\circ}$ 6)

40 À deux reprises le chemin de Pasteur est passé par l'école, toujours ces rencontres furent cruciales. Au sein de cet établissement, Pasteur a tour à tour été élève, enseignant (en qualité d'agrégé préparateur), administrateur, chercheur. 


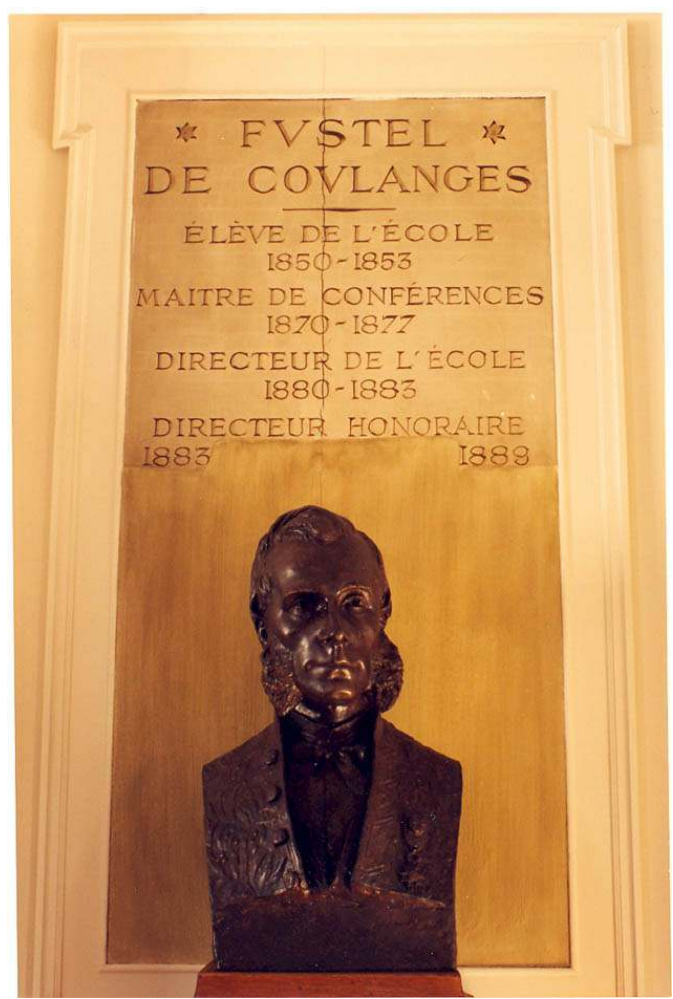

Le buste de Fustel de Coulanges à l'École normale supérieure. Phot. C. Hottin.

(c) Coll. part.

En tant qu'administrateur, Pasteur est également un acteur important de l'histoire de l'institution normalienne au XIXe siècle. Acteur peu aimé au reste, comme le furent d'autres administrateurs après lui : que l'on songe à Lavisse (honni par Péguy), à Gustave Lanson (« l'École normale est un lieu ou Lanson nuit »), ou à Robert Flacelière ${ }^{22}$. Admiré pour son œuvre, il ne l'est guère pour ces qualités humaines, contrairement à tant d'autres figures "familiales ", comme purent l'être le secrétaire général Dupuy ou le bibliothécaire Lucien Herr. Il laisse aux normaliens de son temps le souvenir d'un fonctionnaire rigoureux, sévère, tatillon ${ }^{23}$. 
Figure 8

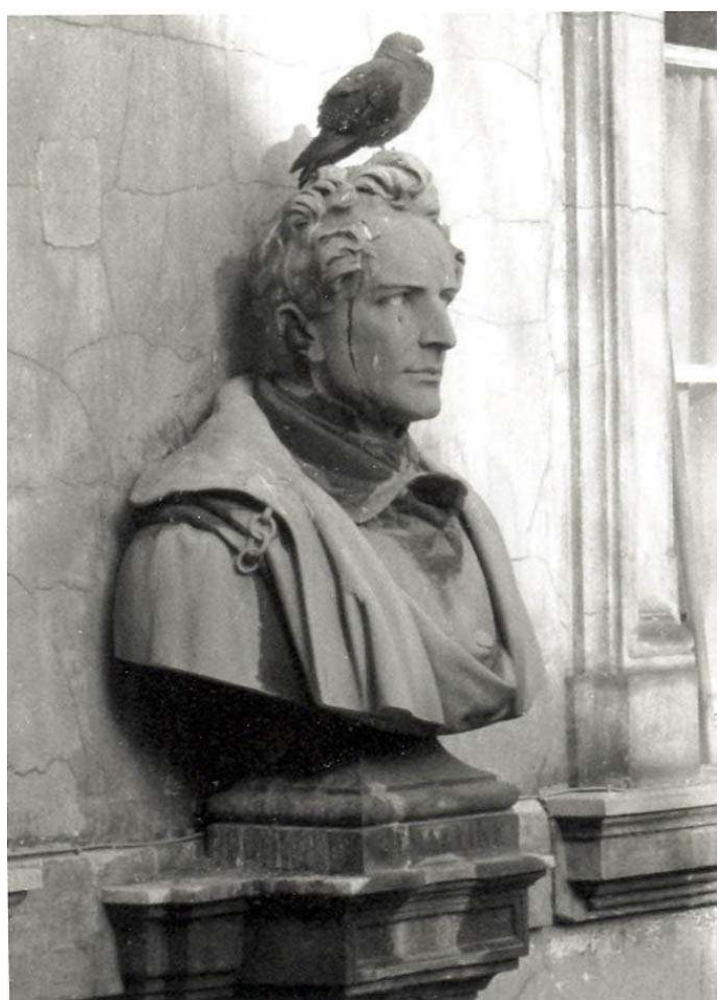

Le buste de Lamartine dans la cour de l'École normale supérieure. Phot. ENS.

Figure 9

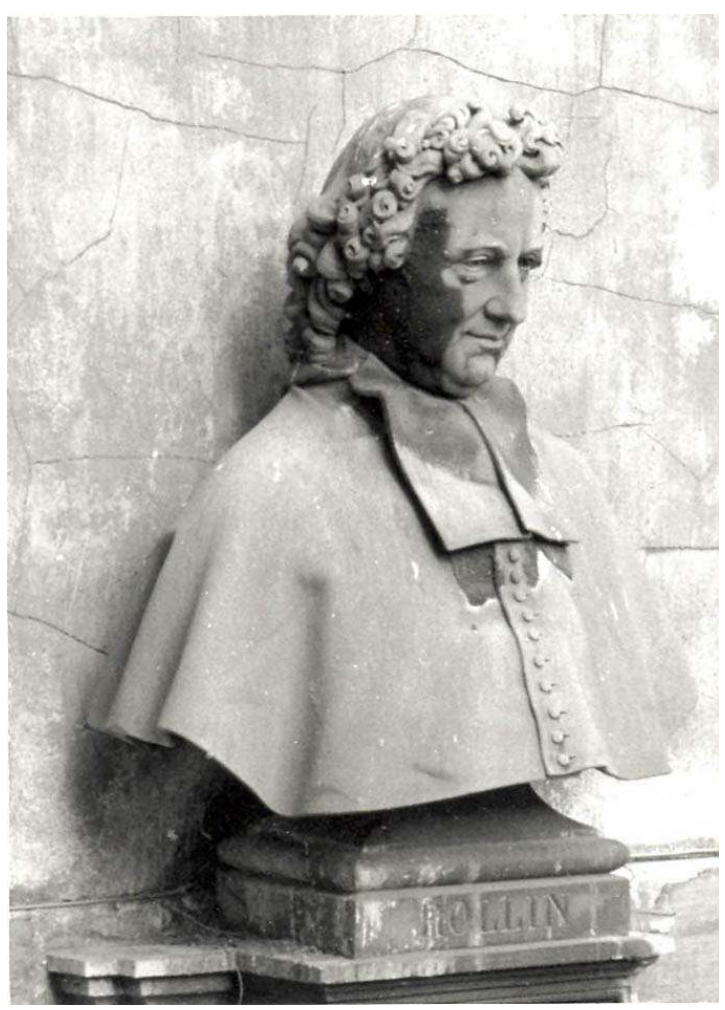

Le buste de Rollin dans la cour de l'École normale supérieure. Phot. ENS. 
Cependant, la figure de Pasteur, par ces multiples occurrences, occupe une place à part dans la décoration de l'école ${ }^{24}$. Celle-ci, du reste, ne recèle en ses murs que peu de figures de normaliens, en dehors des bustes de Fustel de Coulanges et Lucien Herr (fig. $\mathbf{n}^{\circ} \mathbf{7}$ ). L'ensemble d'œuvres le plus important est constitué par les fameux quarante bustes de grands hommes entourant les galeries de la cour principale (fig. $\mathbf{n}^{\circ} \mathbf{8}, \mathbf{n}^{\circ} \mathbf{9}$ ), et le monument le plus remarquable reste sans aucun doute le monument aux morts conçu par Paul Landowski ${ }^{25}$ (fig. $\left.\mathbf{n}^{\circ} \mathbf{1 0}\right)$.

Figure 10

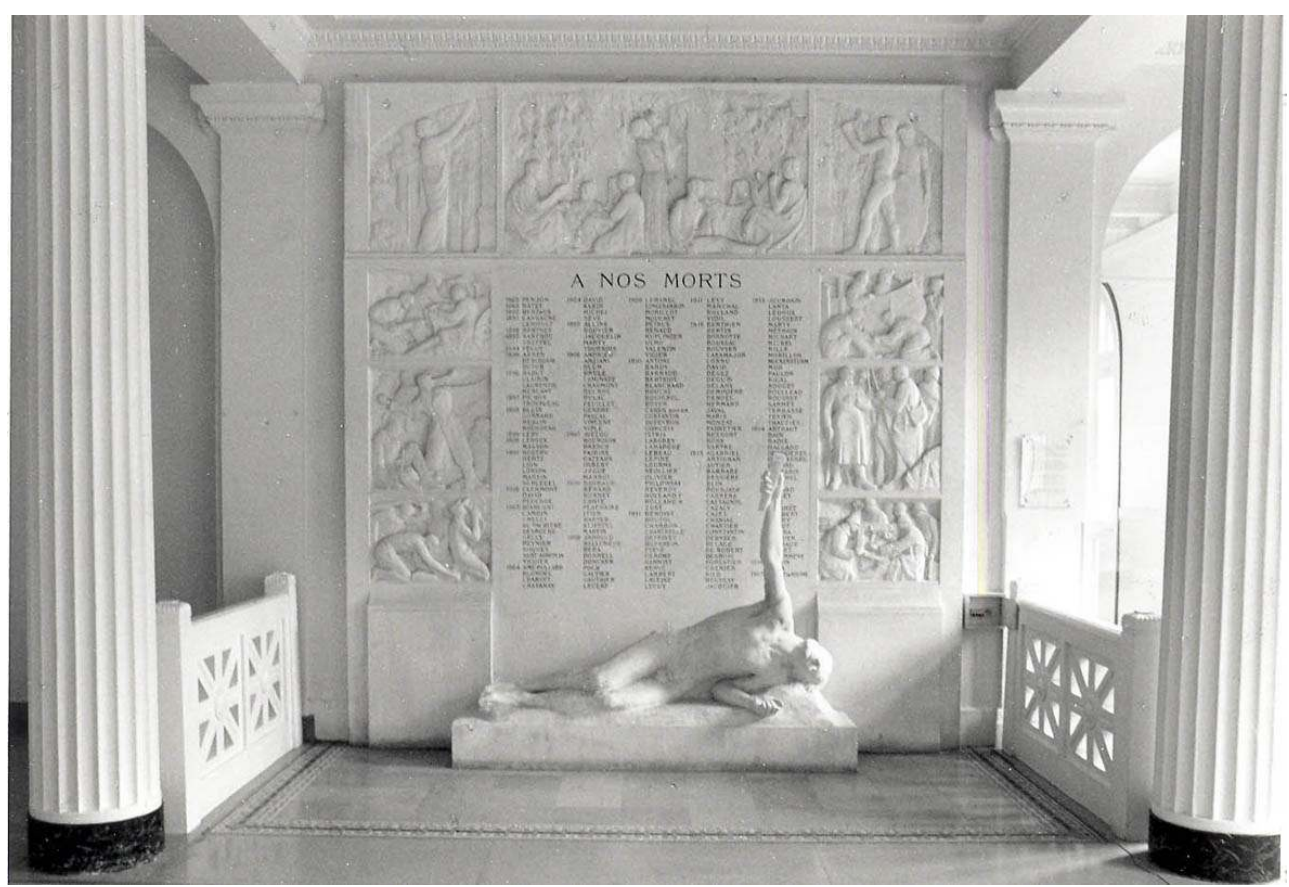

Le monument aux morts de l'École normale supérieure. Phot. ENS.

\section{Le médaillon et les plaques dédiées à Louis Pasteur sur le mur de son laboratoire de l'École normale}

Ce médaillon, fixé sur le mur extérieur du laboratoire, au commencement de la rue d'Ulm, surmonte une plaque de marbre noir portant l'inscription: "Ici fut le laboratoire de Pasteur ». Cette phrase est suivie de la liste des travaux conduits par le chimiste dans ce local. Une petite plaque indépendante indique les dates extrêmes de la présence de Pasteur dans ces murs (1864-1888). L'ensemble fut apposé à la suite d'une délibération du conseil municipal de Paris du 7 décembre $1894^{26}$ (fig. $\mathbf{n}^{\circ}$ 11). 


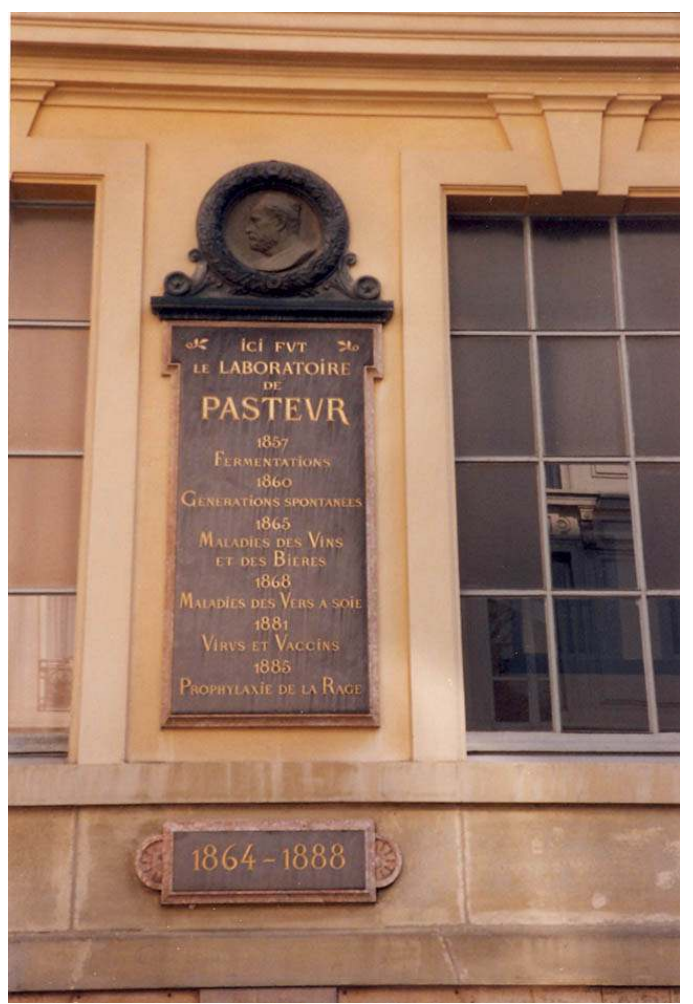

La plaque commémorative des travaux de Louis Pasteur sur la rue d'Ulm. Phot. C. Hottin.

(c) Coll. part.

L'esprit qui présida à l'apposition de ce petit monument est fort différent de celui des deux plaques fixées sur le mur intérieur du laboratoire et qui regardent vers la cour de l'école : la première renseigne sur les installations successives du laboratoire de Pasteur à l'intérieur de l'établissement et sur les agrandissements successifs du local. La seconde donne la liste des principaux collaborateurs de Pasteur à l'École normale.

Il n'est pas question ici de renseigner celui qui pourrait passer devant ce haut lieu en méconnaissant son noble passé : ses plaques sont entièrement consacrées aux relations de Pasteur (en tant que normalien et chercheur) avec son établissement d'origine. Elles s'adressent donc aux initiés ou aux visiteurs avertis susceptibles de parfaire leur connaissance de la geste pastorienne. La différence de mode de financement entre les deux monuments (financement par la Ville pour la plaque installée à l'extérieur, financement probable par l'école pour celles qui se trouvent à l'intérieur) rendent bien compte de ces deux destinations diamétralement opposées.

\section{Le séjour de Pasteur à l'École normale supérieure ${ }^{27}$, par Louis- Édouard Fournier, ou La science et l'humanité}

(fig. $\left.n^{\circ} 12, n^{\circ} 13, n^{\circ} 14\right)$ 
Figure 12

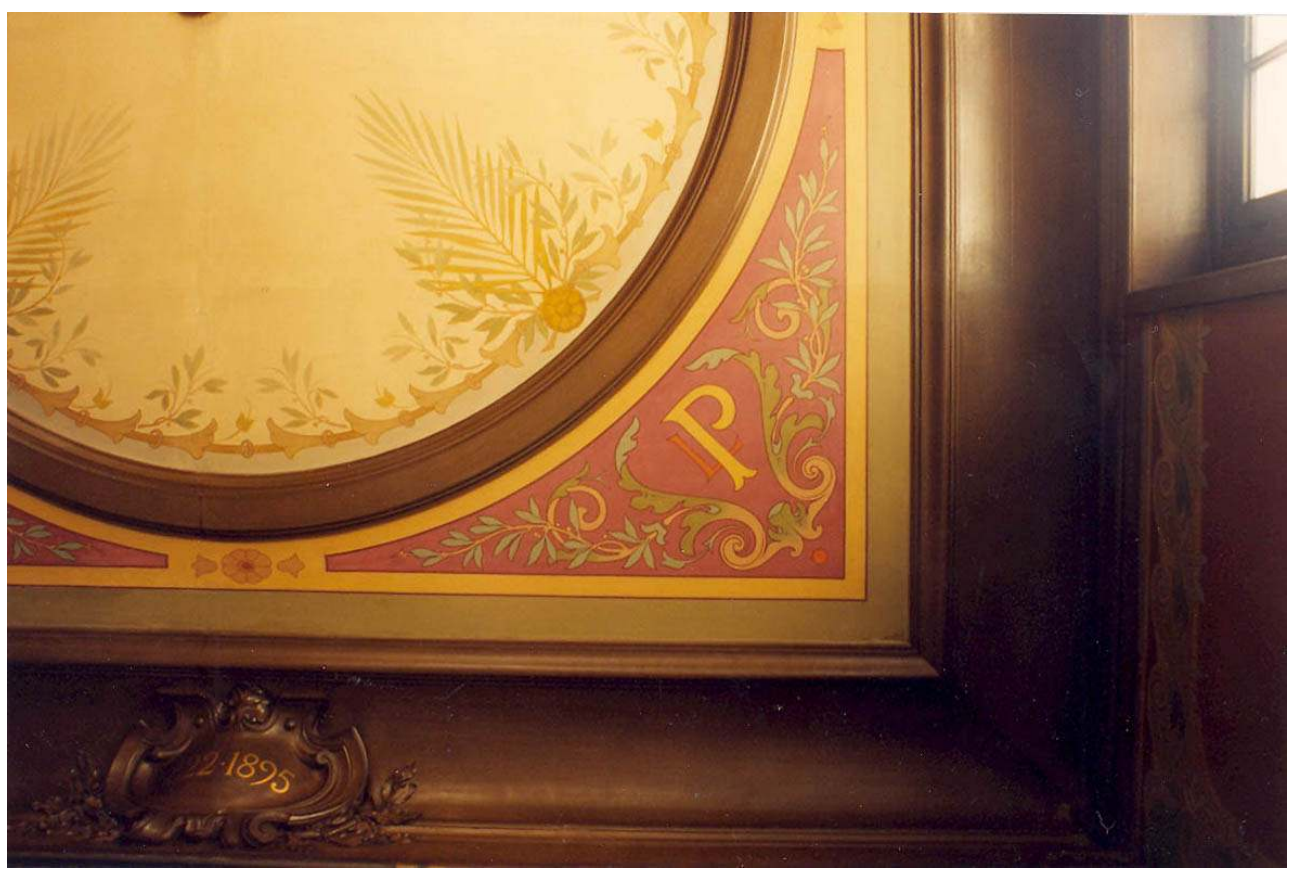

Le séjour de Pasteur à l'École normale supérieure par Louis-Édouard Fournier ou La Science et l'Humanité. Plafond. Phot. P. Reix.

Figure 13

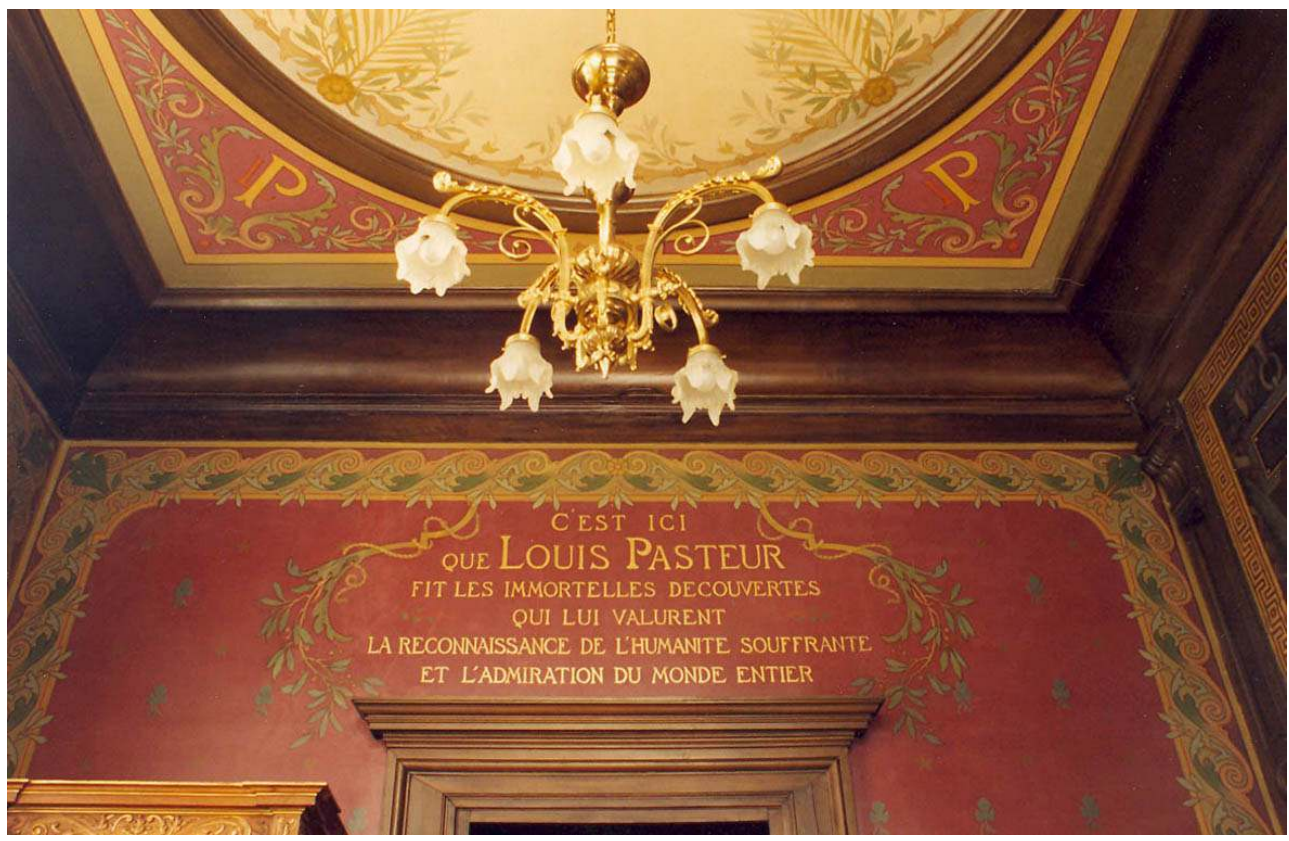

Le séjour de Pasteur à l'École normale supérieure par Louis-Édouard Fournier ou La Science et l'Humanité. Frise. Phot. P. Reix.

L'initiative de cette commande, passée le 2 mars 1895 pour la somme de 2000 francs, revient au directeur de l'École normale supérieure. Ce dernier, après la transformation des laboratoires de Pasteur en infirmerie, souhaita rappeler le souvenir du chimiste sur les lieux même de ses découvertes, dans le bureau de son ancien laboratoire ${ }^{28}$. On confia 
le travail à Louis-Édouard Fournier, premier Grand Prix de Rome en 1881 et ami de la famille de Pasteur. Les travaux furent rapidement menés à leur terme : le dernier examen par un inspecteur des Beaux-Arts eut lieu le 6 avril 1896. La toile fut livrée le 18 avril de la même année.

Figure 14

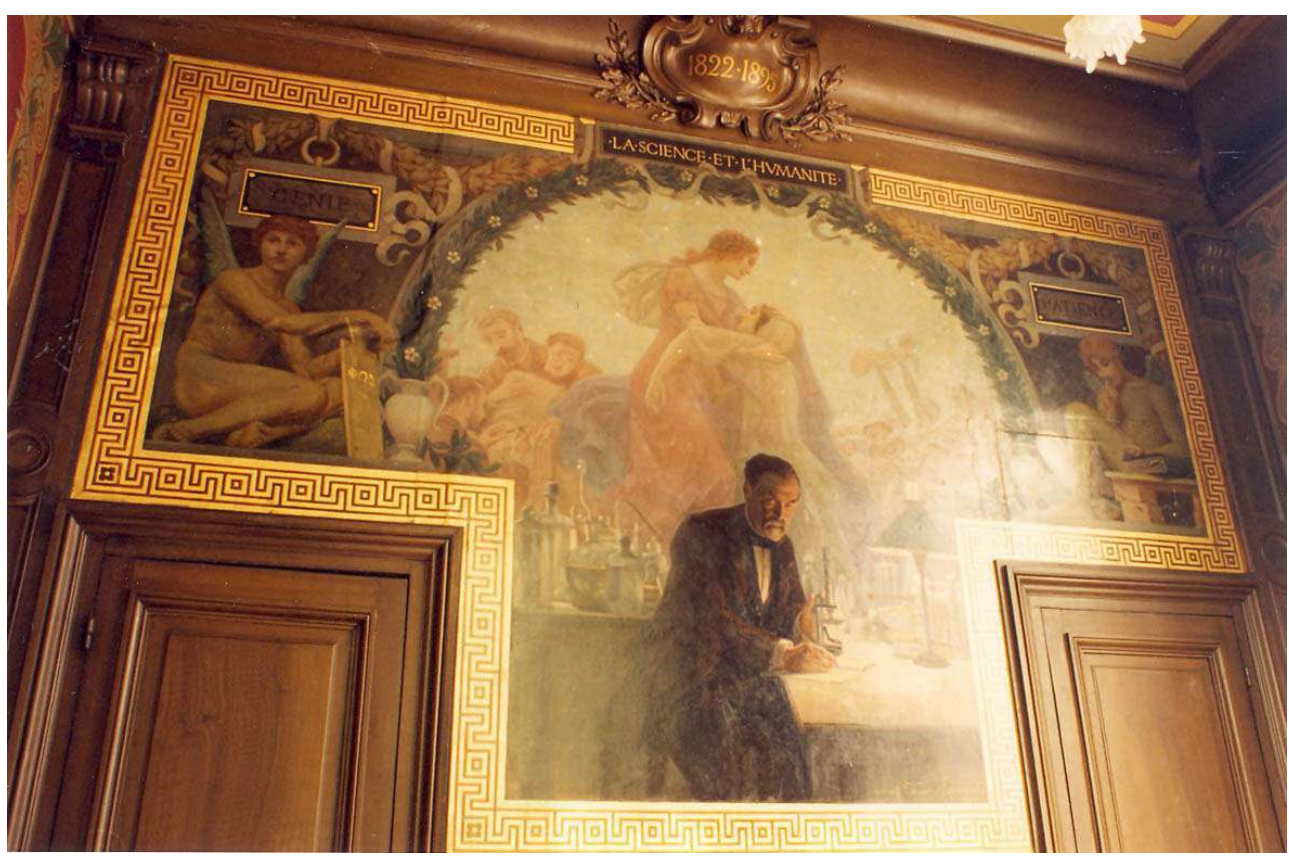

Le séjour de Pasteur à l'École normale supérieure par Louis-Édouard Fournier ou La Science et I'Humanité. L. É. Fournier. Phot. P. Reix.

dant longtemps ces lieux conservèrent leur fonction médicale. Le manque de place dans les vieux locaux de la rue d'Ulm contraignit ensuite l'administration à y installer la section de philosophie. Pendant ce temps, les peintures pâtissaient gravement du chauffage au charbon puis au fioul. Elles s'écaillèrent, les inscriptions devinrent illisibles, la lecture et la compréhension de l'œuvre elles-mêmes devenaient difficiles.

En 1994, année de bicentenaire de l'ENS, on décida de restaurer cet ensemble. Les travaux furent financés par l'École normale supérieure et l'Institut Pasteur avec le soutien du ministère de la Culture. La restauration fut menée à bien par Anne Faiton, Hugues Faideau et leurs collaborateurs. Le bureau restauré fut inauguré le 14 mars 1995 par Simone Veil, alors ministre de la Santé. On apposa alors une plaque commémorative de l'événement à l'entrée de la pièce et on plaça un livre d'or dans le bureau pour permettre aux visiteurs de noter leurs impressions. Cette pièce n'eut désormais plus de fonctions administrative ou universitaire, elle devint un pur et simple lieu de mémoire ${ }^{29}$.

Au centre de la composition, Pasteur apparaît, au soir de sa vie, accoudé à sa table de travail, entouré d'instruments destinés à ses expériences. L'attitude du savant, montré ici en pleine activité, est une représentation fréquente à cette époque (que l'on songe aux œuvres de Léon Lhermitte montrant Claude Bernard ou Henri de Sainte-Claire Deville pour la Sorbonne $)^{30}$. Cependant, à la représentation de l'homme de science entouré de ses pairs et collaborateurs, on a souvent préféré, pour Pasteur, l'exaltation de travail solitaire, en laboratoire, auprès d'une lampe allumée qui suggère de longues et pénibles heures de labeur. La toile marouflée de Fournier s'inscrit dans cette tradition. Elle est 
d'autant moins originale qu'elle s'inscrit nettement dans l'esprit de la célèbre toile d'Edelfelt Pasteur dans son laboratoire.

51 L'originalité de la composition de Fournier réside plutôt dans l'arrière-plan du tableau : Edelfelt nous montre le travail du savant, tandis que Fournier s'attache à rendre visible les résultats de cette activité, au besoin en usant du symbole et de l'allégorie. La mère défaillante soutenue par l'Espoir ailé, les jeunes femmes hissant leurs bébés vers le ciel : toutes ces évocations des bienfaits de l'œuvre pastorienne annoncent les représentations de Pasteur en sauveur du genre humain, et notamment de la famille, ce pilier de la société. Ce thème sera magistralement traité dans le monument de l'avenue de Breteuil : l'enfant est sain et sauf, la mère rassérénée, la Mort hideuse forcée à la retraite. Outre Pasteur, la composition nous donne à voir, dans un coin séparé, le docteur Roux faisant une injection de sérum antirabique à un jeune garçon ${ }^{31}$. Ces scènes s'accompagnent de deux allégories ailées qui encadrent la composition et figurent les qualités principales de Pasteur: le Génie (qui tient une inscription en caractères grecs «phos", c'est-à-dire la lumière) et la Patience.

52 À cette double lecture possible de l'œuvre (historique et documentaire, allégorique et symbolique), s'ajoute une sacralisation de l'ensemble de la pièce qui prolonge la composition de Fournier: le bureau entier est transformé en sanctuaire à la gloire de Pasteur. Ce sont les peintures décoratives qui contribuent à plonger le visiteur dans une ambiance religieuse et quasiment mystique, annonciatrice elle aussi de l'ambiance si particulière du caveau abritant le tombeau de Pasteur. Les initiales entrelacées du grand homme sont peintes sur les quatre coins du plafond, ses dates sont portées au-dessus de l'œuvre et l'on peut lire sur la porte ces mots :

"C'est ici que Louis Pasteur fit les immortelles découvertes qui lui valurent la reconnaissance de l'humanité souffrante et l'admiration du monde entier. »

53 La débauche de dorures et le style quelque peu exalté de ce petit texte nous rapprochent du climat créé par la tombe du savant. Comme on le voit, la commémoration de Pasteur échappe totalement, par son ampleur et par les formes qu'elle prend, à l'univers traditionnel des représentations universitaires.

\section{Le monument à Pasteur de l'École normale supérieure ${ }^{32}$}

(fig. $\left.n^{\circ} 15, n^{\circ} 16\right)$ 
Figure 15

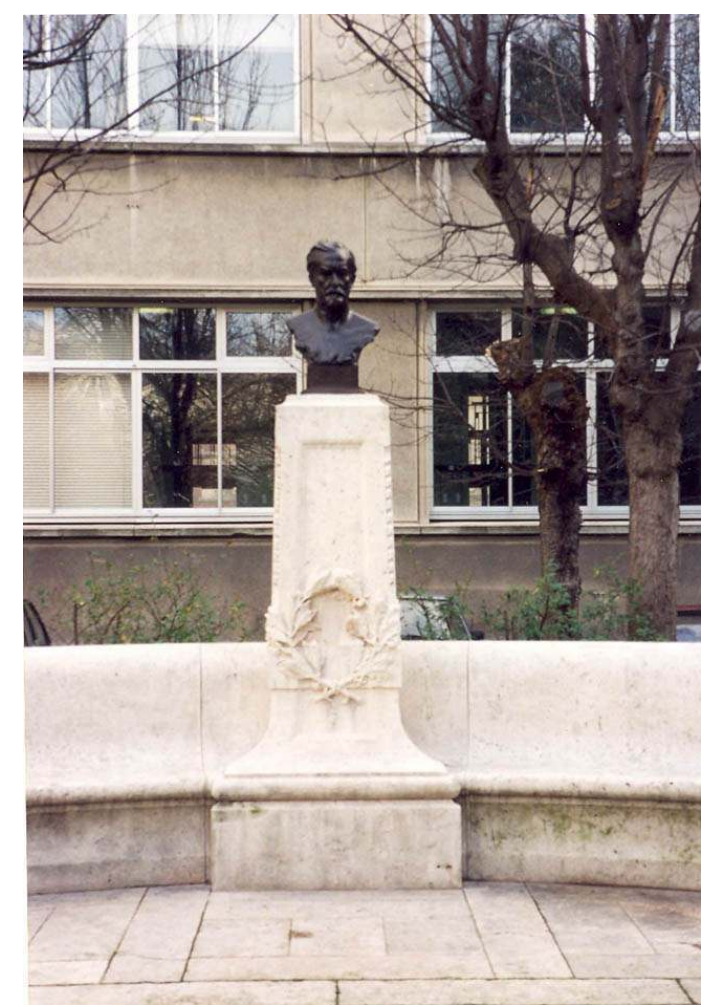

Le monument à Louis Pasteur à l'École normale supérieure. Phot. C. Hottin. (c) Coll. part. 
Figure 16

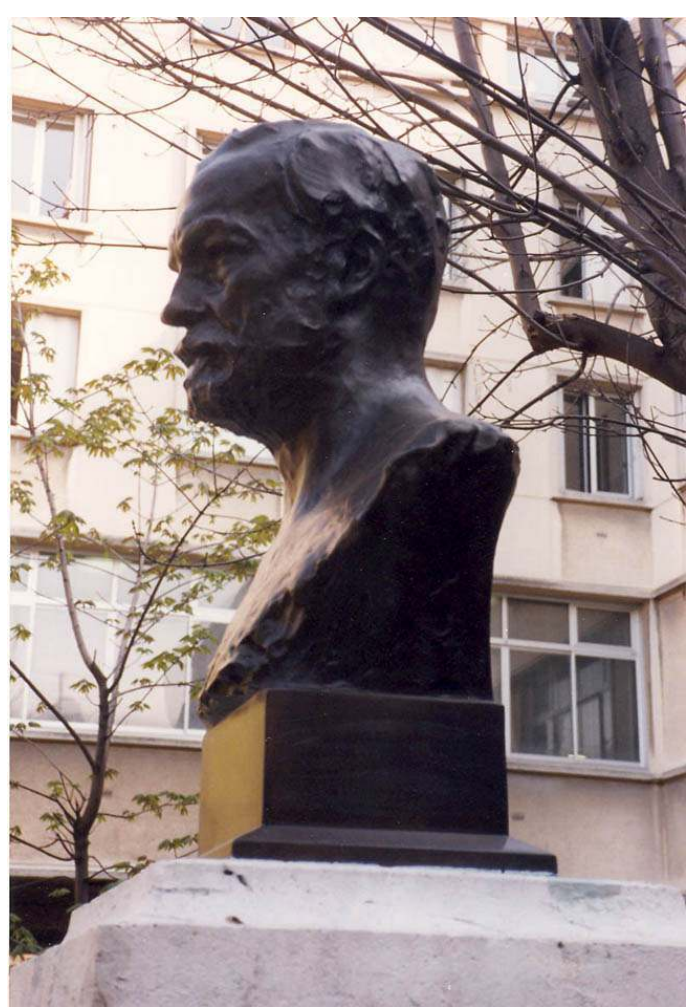

La tête de Pasteur, d'après Dubois, sur son monument à l'École normale supérieure. Phot. C. Hottin.

(c) Coll. part.

En 1909, une colossale souscription internationale permet d'ériger à la gloire de Pasteur le monument conçu par Falguière et installé avenue de Breteuil ; une fois le monument inauguré subsiste un reliquat de 20000 francs. C'est l'architecte Girault qui propose au directeur des Beaux-Arts d'utiliser cette somme pour honorer la mémoire de Pasteur à l'École normale: «Le comité a pensé que nul emplacement n'était mieux à même de consacrer le souvenir du maître que les jardins de l'École normale supérieure qui a été pendant de si longues années le théâtre de ses recherches " ${ }^{33}$.

Une fois la décision prise, les travaux sont conduits rapidement et le monument est inauguré le 6 octobre 1910. Il est présenté comme un don du comité Pasteur: le financement de l'œuvre échappe donc aussi bien à l'administration des Beaux-Arts qu'à la communauté des normaliens. Lors de la cérémonie d'inauguration, c'est le président du comité Pasteur, Darboux, qui remet l'œuvre aux autorités de l'École normale. Par ce geste, il exprime l'hommage de la société à Pasteur et, à travers lui, à l'institution qui l'a formé, qu'il a dirigée, et qui fut le cadre de ses principales découvertes. Le dialogue entre la société et l'institution se substitue ici au dialogue traditionnel de l'institution avec ellemême, qui est la règle pour les monuments élevés par souscription.

En 1957, le buste de Pasteur fut dérobé par les polytechniciens, en représailles au vol du Foch de leur école par les normaliens ${ }^{34}$.

Par sa hauteur et par son importante emprise au sol, cette œuvre se démarque de la plupart des sculptures installées dans les établissements universitaires. La forme générale elle-même est celle d'un monument urbain de petites dimensions, comme on en rencontre dans les squares. Le buste est placé au sommet d'une stèle sur laquelle apparait 
en bas-relief une couronne de lauriers. De part et d'autres de cette stèle un banc de pierre décrit un hémicycle.

Ce monument à Pasteur est le seul présent à l'École normale à être situé en dehors du périmètre sacralisé de son ancien laboratoire. Cependant, comme pour les autres, et son financement le prouve, c'est le souvenir de l'homme de science qu'il honore, et non celui du directeur des études scientifiques de l'école, dont l'action au sein de l'établissement fut, on le sait par ailleurs, diversement appréciée.

L'œuvre semble avoir acquis une certaine place dans la vie normalienne, comme en témoigne une photographie de l'entre-deux-guerres, où l'on voit le buste coiffé d'un calot militaire (manière pour les normaliens de protester contre la préparation militaire obligatoire), tandis que sur la stèle sont massés des jeunes gens, parmi lesquels on reconnaît Louis Poirier, futur Julien Gracq ${ }^{35}$.

\section{Pasteur à la Sorbonne : une icône de la science (française)}

61 Quoiqu'il y ait professé, la Sorbonne (et plus précisément la faculté des sciences) est loin d'être l'établissement qui occupe la place la plus importante dans la vie du savant. S'y trouvent cependant deux représentations de notre héros, qui peuvent être analysées comme deux figurations d'un personnage d'exception en un lieu symbole de l'excellence scientifique française : peu importe alors la proximité effective de l'individu représenté avec l'institution qui accueille son image.

\section{L'édifice et son décor}

On ne reviendra que très brièvement sur le contexte institutionnel et architectural dans lequel s'inscrivent la toile de Rixens et la sculpture de Hugues. Bornons-nous à rappeler l'importance de ce chantier ${ }^{36}$ (le plus considérable entrepris sous la Troisième République pour un édifice d'enseignement supérieur), la qualité du projet de Nénot (notamment en ce qui concerne les équipements scientifiques, et ce en dépit d'un cahier des charges exigeant et d'une limitation de surface contraignante), sa valeur symbolique (la reconstruction de la Sorbonne a une valeur exemplaire pour le développement et la rénovation de l'ensemble du système d'enseignement supérieur, elle accompagne le processus aboutissant en 1896 à la recréation des universités dans notre pays).

63 Tout autant que l'architecture, le décor peint et sculpté porte ce discours. Les deux œuvres ici étudiées sont à ce titre exemplaires.

\section{Le « Jubilé » de Pasteur par Jean-André Rixens ${ }^{37}$}

Commandé le 29 juillet 1893 pour la somme de 10000 francs, le tableau ne fut livré qu'en 1902 ; la commande avait été entre-temps portée à 18000 francs $^{38}$.

Rixens évoque dans cette œuvre le jubilé de Pasteur, événement célébré le 27 décembre 1892. Dans le grand amphithéâtre flambant neuf se pressent les personnalités du monde scientifique européen (professeurs français et étrangers en robe de cérémonie ou en habit), hommes politiques, membres de la famille de Pasteur. Louis Pasteur s'avance au bras du président Sadi Carnot. Le professeur Lister, représentant de la Société royale de 
Londres vient à sa rencontre, les bras tendus. On reconnaît plusieurs éléments de la décoration d'origine : la statue de Richelieu et celle de Pascal, Le Bois sacré de Puvis de Chavannes, le buste de la République (une simple Cérès enlevée depuis).

On peut reprendre brièvement certains éléments de la description donnée par Bonnerot pour se faire une idée plus précise de la composition :

«Le Président de la République Sadi Carnot entre donnant le bras à Pasteur. Tous deux portent le grand cordon de la Légion d'honneur. Lord Lister, le célèbre chirurgien anglais qui représente les sociétés royales de Londres et d'Edimbourg gravit les marches, les mains tendues vers Pasteur, et l'on sait que ce fut une minute émouvante lorsque les deux savants s'embrassèrent aux applaudissements de tous, symbole vivant de la fraternité de la Science dans le soulagement de l'Humanité. (...) ce tableau compte 408 personnages qui sont tous des portraits de personnalités du monde des sciences des arts ou des lettres, ou de la famille de Pasteur ou du peintre. Grâce au schéma qu'en a dressé le Dr Raphaël Blanchard et dont un exemplaire encadré se trouve dans la salle des actes, il est permis de les identifier aisément. C'est un tableau historique reconstitué et nombre des personnes qui y figurent n'ont pas assisté à la cérémonie du 27 décembre 1892 ; la ressemblance des portraits en fait un document précieux. $»^{39}$

Par l'inclusion de cette œuvre quelque peu laborieuse dans le programme décoratif de la Sorbonne, les concepteurs de l'ensemble du projet insèrent un épisode particulièrement glorieux de la vie de Pasteur (la consécration de sa carrière de savant et son apothéose internationale) dans la série des grandes et riches heures de l'histoire de la Sorbonne. Non loin de l'œuvre de Rixens se trouvent en effet les cycles de peintures marouflées de François Flameng et Théobald Chartran, consacrés à l'histoire des lettres et à celle des sciences $^{40}$. Ailleurs dans l'édifice se trouvaient à l'époque de sa reconstruction d'autres œuvres, illustrant des épisodes glorieux de l'histoire de la Sorbonne au XIXe siècle: Quinet et Michelet par Brouillet ${ }^{41}$, Claude Bernard ${ }^{42}$ ou Sainte-Claire Deville ${ }^{43}$ par Lhermitte. Enfin, on peut rapprocher cette évocation du jubilé de Pasteur d'autres décors retraçant quelques-unes des étapes de la renaissance de la Sorbonne à travers sa reconstruction: Signature des plans de la nouvelle Sorbonne par André Brouillet ${ }^{44}$, Pose de la première pierre de la nouvelle Sorbonne par Wencker ${ }^{45}$, Réunion de la Sorbonne et de l'École normale supérieure par Devambez ${ }^{46}$.

Ces points de comparaison soulignent l'importance de Pasteur, tout autant que l'importance de la célébration de ce savant dans le grand amphithéâtre, alors tout récent, de l'édifice qui est présenté comme le cœur vivant de la science française : il abrite en effet aussi bien la faculté des sciences que les services du rectorat.

\section{La statue de Pasteur par Hugues}

Tout comme celle de Victor Hugo, qui lui fait pendant, cette statue n'était pas prévue dans le programme initial de décoration de la cour d'honneur. Nénot proposa son exécution dans un rapport adressé à la direction administrative des services municipaux d'architecture le 23 juin 1899. Ces deux œuvres devaient constituer l'équivalent moderne d'Homère chantant et Archimède installés dans le grand vestibule de la rue des Écoles ${ }^{47}$.

Pasteur fut commandé à Hugues par arrêté du 18 décembre 1899, pour la somme globale de 14000 francs (modèle : 3000 francs, traduction : 11000 francs) $^{48}$. 
71 Le savant tient à la main une cornue, endommagée en 1968 lorsque la statue fut recouverte de banderoles et servit de hampe à de multiples drapeaux rouges.

Comme on le voit, à travers la présence de Pasteur dans le programme décoratif sculpté de la Sorbonne, il s'agit moins de rendre hommage à l'œuvre du savant que de se saisir de cette figure comme d'un emblème, celui des sciences, et de l'inclure dans un système de parallélismes et d'oppositions qui structure tout l'édifice, celui de la dichotomie entre lettres et sciences. Exprimé au moyen de figures de l'Antiquité dans le grand vestibule (Homère par Delaplanche ${ }^{49}$ et Archimède par Falguière ${ }^{50}$ ), il est développé sur le mode de la grande composition historique dans le péristyle (histoire des lettres et histoire des sciences), traité par l'allégorie dans la salle des autorités (statues des Lettres et des Sciences, cycles des peintures d'Ernest Laurent ${ }^{51}$ et Hélène Dufau $\left.{ }^{52}\right)$. On retrouve donc le thème dans le décor de la cour d'honneur. Manque cependant la clef qui permet de réunir ces deux ensembles de disciplines qui paraissent constamment mises en opposition : il s'agit de la grande statue de la Pensée sorbonnienne, voulue par Nénot pour compléter la mise en scène de la partie haute de la cour. Son exécution fut confiée à Denys Puech, mais l'œuvre ne trouva jamais sa place ${ }^{53}$.

\section{Pasteur absent ? La faculté de médecine de Paris}

Figure 17

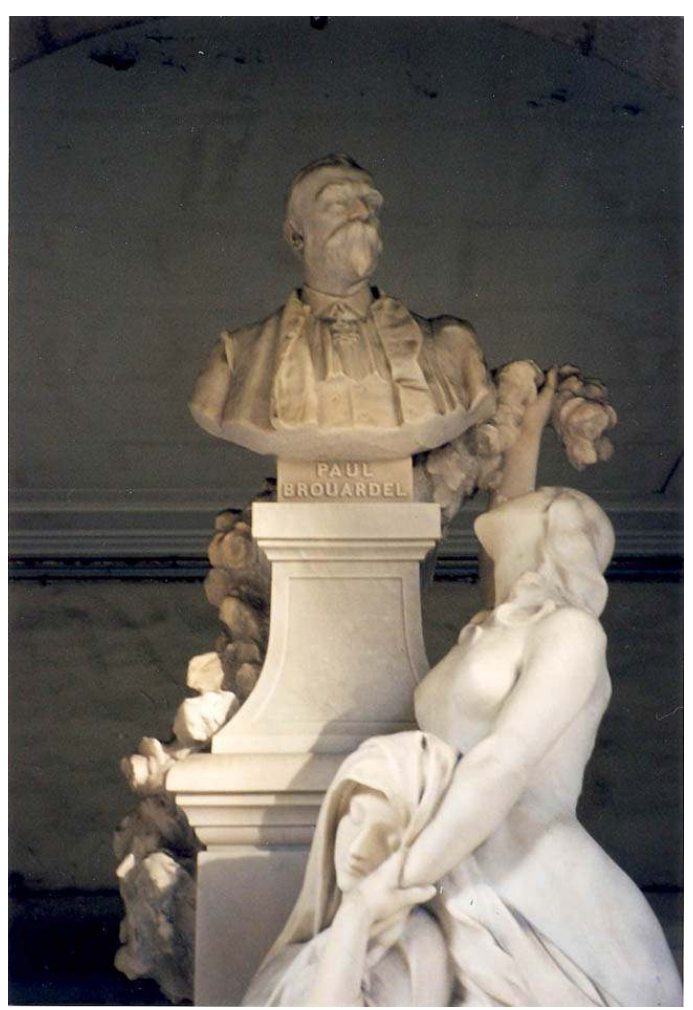

Un exemple de monument élevé par souscription à la Faculté de médecine de Paris. Phot. C. Hottin (c) Coll. part.

Pour qui s'intéresse aux œuvres d'art du monde universitaire, la faculté de médecine occupe une place singulière dans le paysage parisien. En effet, contrairement à la plupart des autres établissements, celui-ci possède des collections de peintures ou de sculptures 
non seulement importantes, mais encore souvent anciennes. Qui plus est, ces œuvres se distinguent de celles conservées dans les autres institutions par la proportion très importantes de dons, legs, ou souscriptions quant au mode d'entrée dans les collections (fig. $\mathbf{n}^{\circ}$ 17). Ce phénomène remonte à la fin de l'Ancien Régime, comme en attestent les bustes les plus anciens, souvent réalisés en vue de décorer l'édifice nouvellement construit par Gondoin pour abriter l'Académie de chirurgie. La tradition s'est perpétuée tout au long du siècle suivant, tandis que plusieurs séries de bustes venaient embellir les espaces de la faculté par le biais de la commande publique. Ce fut le cas notamment lors de la reconstruction presque complète de l'école pratique et de l'agrandissement de la faculté, deux opérations de grande ampleur conduite par Ginain à la fin du XIXe siècle.

Reste que le plus important décor de la faculté réside dans la peinture murale exécutée pour le grand amphithéâtre. Pour ce chef-d'œuvre de l'architecture néoclassique, source d'inspiration considérable pour les bâtisseurs d'amphithéâtres des décennies suivantes ${ }^{54}$, pas moins de trois compositions furent successivement imaginées. La première, œuvre de Gibelin contemporaine de la construction de la salle, donnait à voir une représentation des valeurs du métier de chirurgien dans un cadre antiquisant. La seconde, œuvre de Matout exécutée sous le Second Empire, mettait en scène quelques épisode fameux de l'histoire de la profession. Elle disparut dans un incendie. Les projets esquissés par divers artistes pour la remplacer intéressent directement notre propos.

\section{Pasteur et la médecine : une relation ambiguë}

Si par un rapide sondage on interrogeait un échantillon de personnes sur la profession de Pasteur, il est probable que celui-ci apparaîtrait à beaucoup comme un «médecin ». De fait, si une part importante des travaux de Pasteur a touché de près à la médecine (ou aux sciences vétérinaires), et s'il est vrai également que ses découvertes ont largement contribué à infléchir le cours de la recherche et de la pratique médicale, il n'en reste pas moins que Louis Pasteur n'était médecin ni de formation, ni de métier. Les détails donnés précédemment sur son parcours montrent assez que, bien qu'ayant fréquenté de nombreux établissements au cours de ses études ou de sa carrière, notre héros ne fréquenta guère la faculté de médecine de Paris. Qui plus est, son parcours (normalien et agrégé) diffère totalement de celui de l'élite médicale, généralement issue de l'Internat en médecine, institué par Napoléon pour être un grande école de la pratique médicale: l'essentiel de la formation du médecin doit s'accomplir au sein de l'hôpital, se fortifier par la pratique régulière des dissections, et ne repose que très accessoirement sur les cours reçus en faculté. Si au cours de ses recherches Pasteur a travaillé avec des médecins (ainsi Brouardel l'assista lors de l'expertise de ses vaccins contre la rage) et s'il a contribué à former des disciples qui étaient par ailleurs médecins de formation, il a également rencontré au sein de cette profession des hostilités et des oppositions à ses théories. Oppositions qui semblent avoir été suffisamment fortes pour que lui soit refusé l'honneur d'une représentation picturale sur les murs de la faculté de Paris.

\section{Trois projets, avec ou sans Pasteur, une réalisation, toujours sans lui...}

En 1883, Louis Matout, qui exécuta quelques années auparavant la peinture détruite du grand amphithéâtre, propose un projet de décor pour la salle de la lecture de la future bibliothèque (la faculté est alors en pleine reconstruction). Quoique d'un âge avancé, le peintre ne désarme pas et souhaite visiblement transmettre à la postérité une œuvre pour 
la faculté. Son projet de frise doit se développer sur $90 \mathrm{~m}$ de long et $3 \mathrm{~m}$ de haut. Il s'agit d'une série de représentations des hommes qui ont illustré la médecine depuis l'Antiquité jusqu'à la fin du XIXe siècle ${ }^{55}$. Deux groupes de médecins (les Grecs et les Arabes) occupent les extrémités. Au centre, les médecins et chirurgiens de la Renaissance encadrent, à droite et à gauche, les savants des Temps modernes. On peut noter que si cinq seulement des médecins de la Renaissance sont français (sur vingt-quatre), tous les modernes, sans exception, le sont... Parmi eux, Pasteur, qui clôt la liste, venant ainsi après Bichat, Corvisart, Laënnec, Andral, Velpeau et Claude Bernard.

77 Le projet ne reçut au demeurant aucune suite: il s'agissait d'une initiative toute personnelle de Matout.

78 En revanche, ce dernier obtient en 1884 une commande ferme, d'un montant de 20000 francs, pour la décoration de la salle du conseil du nouvel édifice ${ }^{56}$. Une fois celle-ci exécutée $^{57}$, Matout tenta de relancer le projet de décoration de la bibliothèque. Le programme alors élaboré par l'artiste reprend, dans ses grandes lignes, le projet avorté de 1883. Si les Arabes ont disparu, on y retrouve en revanche les Grecs (groupés sous le titre "Athènes ») et les médecins de la Renaissance, français ou non, et des temps modernes (groupés sous le titre « Paris »). La place faite à Pasteur est ici étonnante : il apparait de nouveau à la dernière ligne de la liste, et Matout, craignant peut-être un refus, a écrit à côté de son nom « si l'on veut $»^{58}$ !

79 Pas plus que le précédent ce projet ne retint l'attention des administrations, qu'il s'agisse de celle des Beaux-Arts ou de celle de la Faculté.

80 Enfin, c'est à un autre peintre, Urbain Bourgeois, qu'échut la commande d'une toile marouflée de grande taille pour l'amphithéâtre. À bien des égards, son « Assemblée des médecins de tous les temps réunis sous la présidence d'Hippocrate » rappelle les projets de Matout. Bourgeois obtint la commande en 1891 et l'œuvre fut installée en 1896. À aucun moment, pour autant que peuvent le laisser supposer les archives du dossier de commande, il ne fut question d'inclure Pasteur dans cette réunion des médecins les plus illustres ${ }^{59}$... Claude Bernard ferme la marche.

81 Quelques décennies plus tard, les médaillons exécutés pour la façade de la nouvelle faculté de médecine, rue des Saints-Pères, font une large place aux médecines grecques et arabes, de même qu'aux savoirs médicinaux des autres civilisations... Mais Pasteur demeure absent de la liste (fig. $\left.n^{\circ} \mathbf{1 8}, n^{\circ} 19\right)$. 
Figure 18

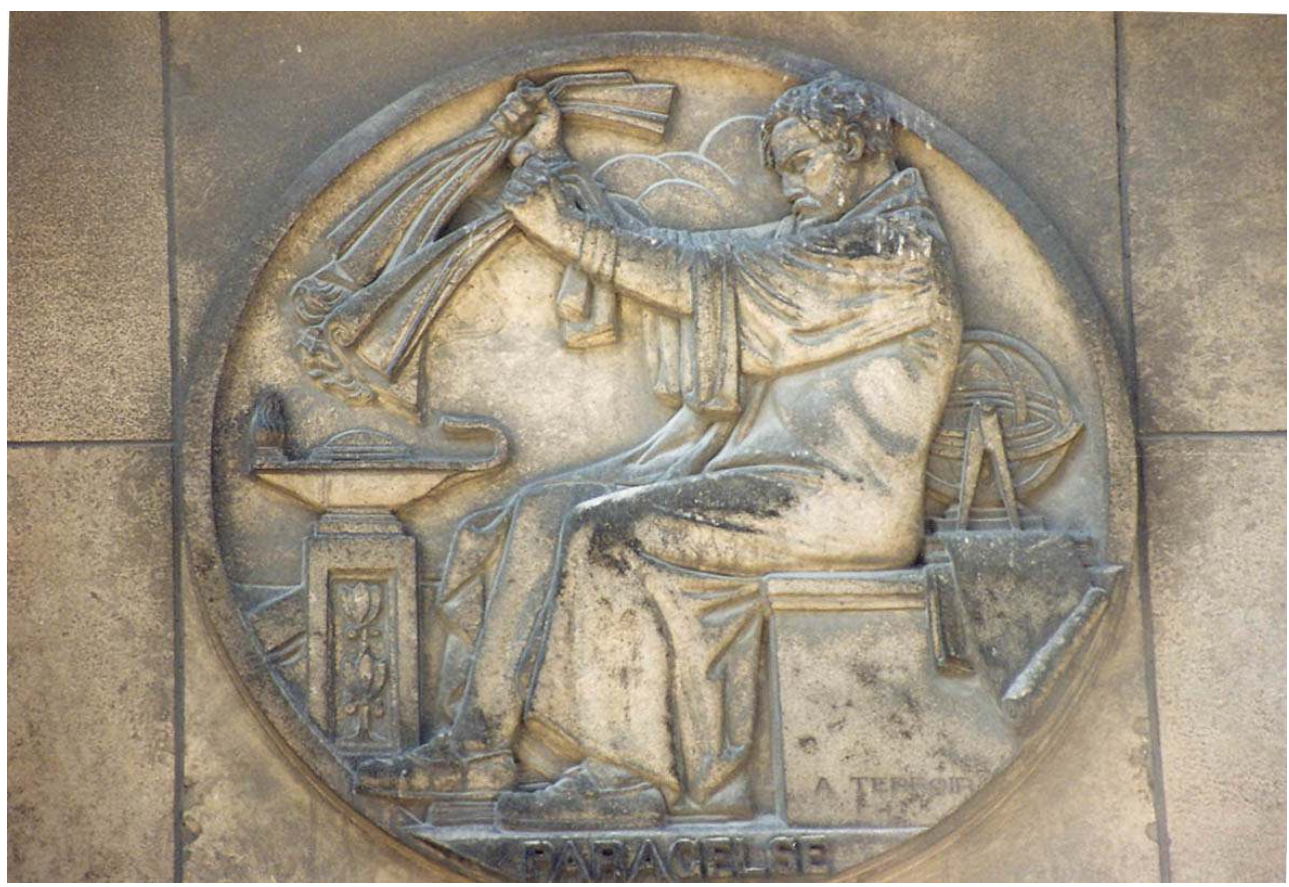

Paracelse brûlant les anciens traités de médecine. Phot. C. Hottin.

(c) Coll. part.

Figure 19

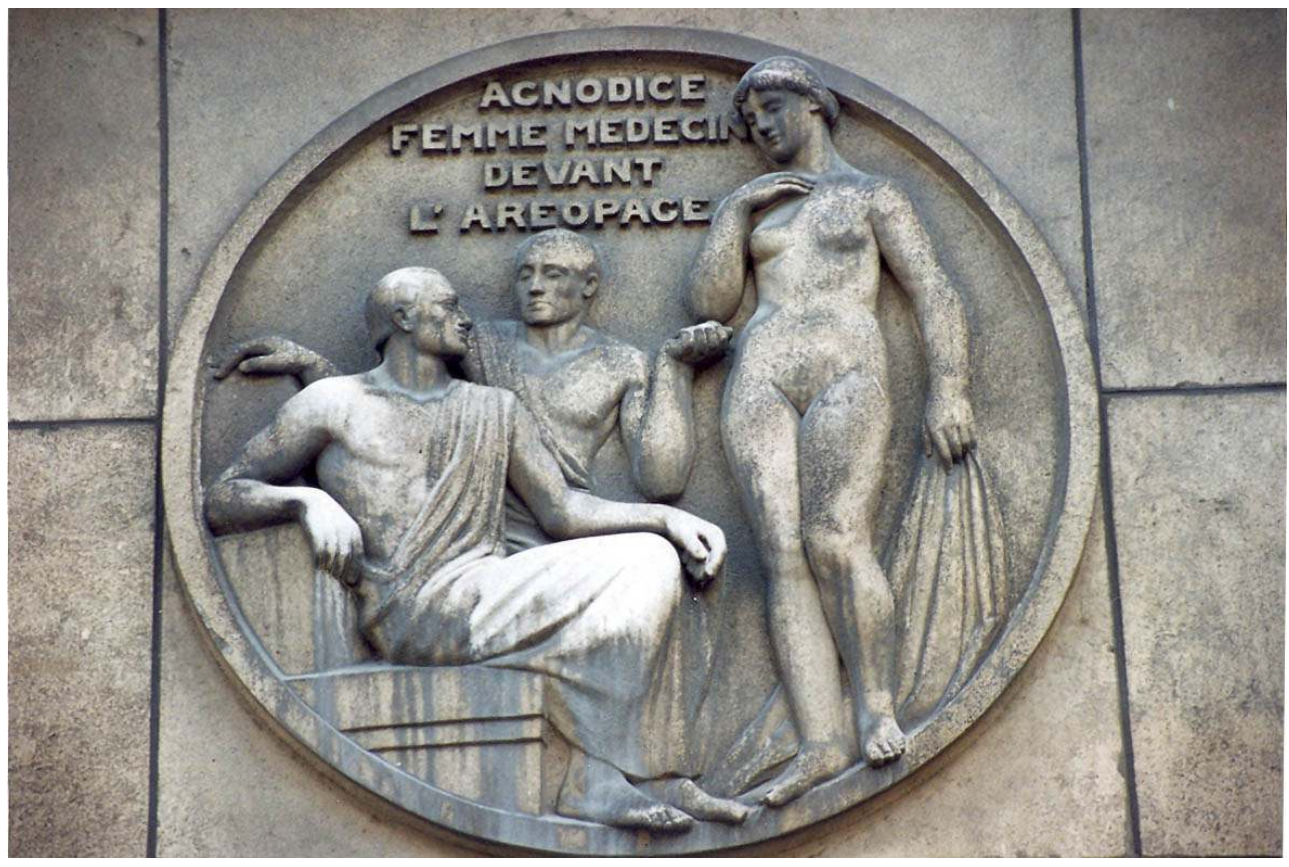

Agnodice, femme médecin devant l'aréopage. Phot. C. Hottin.

(c) Coll. part. 


\section{Conclusion}

82

Lors de la présentation générale de la problématique de cet atelier consacré à la figure de Pasteur en héros, Noël Barbe a proposé quatre déclinaisons de la figure héroïque de Pasteur ${ }^{60}:$ domestique, civique, inspiré et couronné. Cette hypothèse de départ est pour l'essentiel confortée par l'analyse détaillée des représentations pastorienne dans un contexte géographique et institutionnel précis tel que celui des établissements d'enseignement supérieur parisiens.

Héros domestique, Pasteur l'est assurément à travers la multiplicité des représentations le concernant au sein de l'ENS, qui mérite de figurer dans les annales comme la cinquième demeure pastorienne. Héros civique, figure de la communauté nationale : il est également tel, figure incarnant la science française, à l'égal de Victor Hugo dans le domaine des lettres. Héros inspiré : il l'est encore, à travers la figuration du savant au travail, courbé sur sa table d'expériences. Couronné: il l'est enfin, comme en témoigne l'apothéose reconstruite par Rixens dans le tableau de la Sorbonne.

\section{NOTES}

1. Pour une présentation complète du séminaire et de son programme, voir: http:// www.lahic.cnrs.fr/spip.php ?article380

2. Ont pris part à ce séminaire, par ordre d'intervention: Daniel Fabre, Noël Barbe, Christian Amalvi, Annick Perrot, Agnès Desquand, Odile Vincent et Christian Hottin.

3. La thèse a été publiée: HOTTIN, Christian. Quand la Sorbonne était peinte. Paris: Maisonneuve et Larose, 2001. Pour une version du manuscrit disponible en ligne: http:// halshs.archives-ouvertes.fr/halshs-00136677/fr/

4. AGULHON, Maurice (dir.). Culture et folklores républicains, actes du colloque « Les marques républicaines dans la culture populaire en France", Toulouse, 10-12 décembre 1992, Paris: éditions du CTHS, 1995.

5. AGULHON, Maurice. Marianne au combat: l'imagerie et la symbolique républicaines de 1789 à 1880. Paris: Flammarion, 2001 [réed.]; Marianne au pouvoir : l'imagerie et la symbolique républicaines de 1880 à 1914. Paris : Flammarion, 1989 ; Marianne dans la cité. Paris: Imprimerie nationale, 2001; Les métamorphoses de Marianne : l'imagerie et la symbolique républicaines de 1914 à nos jours. Paris : Flammarion, 2001.

6. HARGROVE, June. Les statues de Paris : la représentation des grands hommes dans les rues et sur les places de Paris. Paris : Albin Michel, 1989.

7. Le triomphe des mairies : grands décors républicains à Paris, 1870-1914, catalogue de l'exposition au musée du Petit Palais, 8 novembre 1986-18 janvier 1987. Paris : musée du Petit Palais, 1986.

8. FOUCART, Bruno. Le renouveau de la peinture religieuse en France : 1800-1860. Paris : Arthéna, 1987; LENIAUD, Jean-Michel. Jean-Baptiste Lassus ou le temps retrouvé des cathédrales. Paris : Arts et métiers graphiques, 1980. 
9. On pense ici notamment au sauvetage in extremis des œuvres de René Ménard par les conservateurs du Louvre, peu de temps avant la destruction de la salle des actes de la faculté de droit, œuvre remarquable de Lheureux qui les abritait. Voir : HOTTIN, Christian. Les visages du droit, Bull. de la société d'histoire de l'art français, à paraître en 2009.

10. Le premier travail de grande ampleur sur cette période reste l'ouvrage de Bruno Foucart, issu de sa thèse. Il convient également de signaler l'étude pionnière de Lin Young Bang, datant de la fin des années 1960 et consacrée aux décors peints des édifices publics parisiens au XIXe siècle : Le décor des édifices civils publics à Paris sous la troisième République. Paris, thèse universitaire de lettres, Paris, 1964.

11. BOURDIEU, Pierre. Homo academicus. Paris : Éditions de minuit ; La noblesse d'État. Paris : Éditions de minuit, 1989.

12. Daniel Fabre et Noël Barbe : «Pasteur en héros : l'institution d'un patrimoine scientifique?», séance du 9 janvier 2008.

13. AMALVI, Christian. Les héros de l'histoire de France : recherche iconographique sur le panthéon scolaire de la Troisième République. Paris : Phot-œil, 1979.

14. AVEZOU, Laurent. Sully à travers l'histoire : les avatars d'un mythe politique. Paris : École nationale des chartes, 2001.

15. HOTTIN, Christian. Les collections de bustes, Universités et grandes écoles à Paris : les palais de la Science. Paris : AAVP, 1999, p. 69-75. Une autre version de ce texte est disponible sur le site de l'HAL-SHS : http://halshs.archives-ouvertes.fr/halshs-00088137/fr/

16. Voir : HOTTIN, Christian. Les commandes de peintures, Universités et grandes écoles à Paris : les palais de la Science. Paris : AAVP, 1999, p. 59-69. Une autre version de ce texte est disponible sur le site de l'HAL-SHS : http://halshs.archives-ouvertes.fr/halshs-00088296/fr/ 17. Information communiquée par Ségolène Bergeon Langle.

18. Voir : PASTEUR, Louis. Le budget de la science. Paris : Gauthier-Villars, 1868; Quelques réflexions sur la science en France. Paris : Gauthier-Villars, 1871.

19. Voir : HOTTIN, Christian. La Sorbonne ou l'impossible défi de M. Nénot, La Sorbonne : un musée, ses chefs-d'œuvre (en collab. avec Geneviève Bresc-Bautier, Jacques Foucart, Alexandre Gady, Anne Pingeot et Pierre Rosenberg). Paris: Chancellerie des universités - Réunion des musées nationaux, 2007, p.74-96. Texte disponible sur le site de l'HAL-SHS: http:// halshs.archives-ouvertes.fr/halshs-00121241/fr/

20. Plus généralement, rares sont les personnages qui font l'objet d'autant d'attention au sein d'une même institution. Seuls les maréchaux Joffre et Foch, de multiples fois distingués dans l'iconographie et le décor de l'École polytechnique paraissent s'en approcher.

21. Arch. nat., 61 AJ 163. Érection du monument à Louis Pasteur, discours d'Ernest Lavisse, p. 8.

22. Au début des années 1970, Robert Flacelière, directeur de l'École, fait l'objet d'attaques répétées de la part des activistes gauchiste de la rue d'Ulm, notamment lors du centenaire de la Commune de Paris. Voir: HOTTIN, Christian. 80 ans de la vie d'un monument aux morts, Le monument aux morts de l'École normale supérieure, Labyrinthe, $n^{\circ} 5$, hiver 2000, p. 61-78. Texte disponible en ligne :

http://labyrinthe.revues.org/index262.html\#text.

23. Cette image de lui perdure au XXe siècle, comme en atteste l'insertion dans le recueil d'Alain Peyrefitte d'un rapport hebdomadaire de Pasteur au ministre, sous le titre « un administrateur à poigne ». Le texte débute ainsi : «un grave désordre s'est produit dimanche dans la chapelle. Pendant la lecture d'un mandement de Mgr l'archevêque de Paris, des murmures sont partis des rangs occupés par les élèves de deuxième année (lettres) (...). Ces murmures sensibles, mais peu prononcés au commencement, sont devenus à partir d'un certain moment assez distincts et significatifs pour obliger l'aumônier à s'arrêter et à interpeller les élèves. Moi-même je manifestai mon vif mécontentement, malgré la retenue que m'inspirait l'endroit où nous nous trouvions ». PEYREFITTE, Alain. Rue d'Ulm. Paris : Fayard, 1994, p. 33-34. 
24. Sur l'architecture de l'ENS, voir : BENOIT, Serge. La rue d'Ulm, Universités et grandes écoles à Paris, Paris : AAVP, 1999, p. 177-182.

25. Voir : HOTTIN, Christian. 80 ans de la vie d'un monument aux morts, Le monument aux morts de l'École normale supérieure, Labyrinthe, $\mathrm{n}^{\circ}$ 5, hiver 2000, p. 61-78. Texte disponible en ligne : http://labyrinthe.revues.org/index262.html\#text.

26. La commémoration du centenaire de Pasteur par la Ville de Paris. Paris, 1924, p. 98.

27. CEuvre datée de 1896, huile sur toile marouflée, signée en bas à droite « Louis E. Fournier ».

28. Arch. nat., F 21 2133. Lettre du directeur des Beaux-Arts au directeur de l'Enseignement supérieur.

29. Ces informations nous ont été aimablement communiquées par Serge Benoit en avril 1995.

30. Voir: LE PELLEY-FONTENY, Monique. Léon Lhermitte, catalogue raisonné de l'œuvre peint. Paris : 1991, p. 51 et pl. 18 ; p. 106-107 et pl. 17.

31. La commémoration du centenaire de Pasteur par la Ville de Paris. Paris, 1924, p. 107.

32. CEuvre de Pierre Paulin, sculpteur, et de Girault et Mayeux, architectes, 1910, buste en bronze sur monument en pierre. Le buste actuel est une copie, installée en 1984, inspirée du buste de Dubois, « auquel il faut toujours revenir quand on veut se faire une idée exacte de la physionomie de Pasteur à l'époque de ses grandes découvertes ». La commémoration du centenaire de Pasteur par la Ville de Paris. Paris, 1924, p. 111.

33. Arch. nat., F 21 4850. Lettre de Girault au directeur des Beaux-Arts, datée du 12 juillet 1909.

34. PEYREFITTE, Alain. Rue d'Ulm. Paris : Fayard, 1994, p. 157-158.

35. DUFAY, François. Des normaliens, de Charles Péguy à Bernard-Henri Lévy. Paris, 1994, p. 321.

36. Voir: RIVÉ, Philippe (dir.). La Sorbonne et sa reconstruction. Paris et Lyon: La Manufacture/AAVP, 1987.

37. RIXENS, Jean-André. Le Jubilé de Pasteur,1902, Huile sur toile, 280x545, signé et daté en bas à droite : « A. Rixens 1902 ».

38. Arch. nat., F 21 2047, dossier de commande.

39. BONNEROT, Jean. La Sorbonne, sa vie, son œuvre, son rôle à travers les siècles. Paris : PUF, 1927, p. 157-158. Bonnerot, toujours précis et soucieux d'objectivité ajoute : «Ce tableau au premier coup d'œil semble une banale image coloriée, faute du recul nécessaire. Si l'on veut bien le voir, il faut se mettre entre les deux montants de la porte de la salle, placer ses deux mains à plat l'une au dessus de l'autre à la hauteur des yeux pour ne laisser qu'un rai de lumière et regarder à travers cet interstice. Les personnages se détachent en pleine clarté. ", p. 158.

40. Voir: HOTTIN, Christian. Inventaire du patrimoine monumental peint et sculpté de la Sorbonne : Rectorat, Faculté des lettres, Faculté des sciences, École nationale des chartes, École pratique des hautes études, La Sorbonne, un musée, ses chefs-d'œuvre (en collab. avec Geneviève Bresc-Bautier, Jacques Foucart, Alexandre Gady, Anne Pingeot et Pierre Rosenberg). Paris : Chancellerie des universités - Réunion des musées nationaux, 2007, p. 204-205 et 221-222.

41. Voir: HOTTIN, Christian. Inventaire du patrimoine monumental peint et sculpté de la Sorbonne, op. cit., p. 197.

42. Voir: LE PELLEY-FONTENY, Monique. Léon Lhermitte, catalogue raisonné de l'œuvre peint. Paris : 1991, p. 51 et pl. 18 ; p. 106-107 et pl. 17.

43. Voir : LE PELLEY-FONTENY, Monique. Léon Lhermitte, catalogue raisonné de l'œuvre peint. Paris : 1991, p. 51 et pl. 18 ; p. 106-107 et pl. 17.

44. Voir: HOTTIN, Christian. Inventaire du patrimoine monumental peint et sculpté de la Sorbonne, op. cit., p. 196.

45. Voir: HOTTIN, Christian. Inventaire du patrimoine monumental peint et sculpté de la Sorbonne, op. cit., p. 266.

46. Voir: HOTTIN, Christian. Inventaire du patrimoine monumental peint et sculpté de la Sorbonne, op. cit., p. 211-212. 
47. Arch. Ville de Paris, VM 62 II, dossier de la Sorbonne.

48. Arch. Ville de Paris, registre des Beaux-arts, document non coté, année 1900.

49. HOTTIN, Christian. Inventaire du patrimoine monumental peint et sculpté de la Sorbonne, op. cit., p. 210.

50. HOTTIN, Christian. Inventaire du patrimoine monumental peint et sculpté de la Sorbonne, op. cit., p. 219.

51. HOTTIN, Christian. Inventaire du patrimoine monumental peint et sculpté de la Sorbonne, op. cit., p. 234.

52. HOTTIN, Christian. Inventaire du patrimoine monumental peint et sculpté de la Sorbonne, op. cit., p. 215.

53. Voir : PINGEOT, Anne. Le décor sculpté de la Sorbonne dans la seconde moitié du XIXe siècle, La Sorbonne, un musée, ses chefs-d'œuvre (en collab. avec Geneviève Bresc-Bautier, Jacques Foucart, Alexandre Gady, Anne Pingeot et Pierre Rosenberg). Paris : Chancellerie des universités Réunion des musées nationaux, 2007, p. 134-163.

54. Voir à ce propos les analyses de LAGET, Pierre-Louis. Le premier amphithéâtre d'anatomie de la communauté des chirurgiens de Paris, Chirurgie, 1999, n 124, p. 681-690, p. 688.

55. Arch. nat., F 21 4397. Programme proposé par Louis Matout pour l'exécution des peintures qui doivent décorer la grande frise de la bibliothèque de la faculté de médecine de Paris, projet daté du 16 janvier 1883.

56. Arch. nat., F 212088. Dossier de commande, arrêté du 30 janvier 1884.

57. L'œuvre fut exécutée mais jamais mise en place. Il s'agissait d'une composition tout à la fois mythologique et allégorique. D'une part était figurée une apothéose d'Esculape, de l'autre, une Déesse entourée par les sciences: Histoire naturelle, Botanique, Chimie, Physique et Électricité. Le 5 juillet 1884, le comité des travaux d'art refusa ces esquisses qualifiées « d'une faiblesse sans égal ». Par lettre du 23 août 1885, l'inspecteur des Beaux-Arts Charles Yriarte protesta contre la poursuite de la commande, qualifiant ses esquisses d'« extraordinaires ». L'œuvre achevée partit au dépôt. Arch. nat., F 21 2088. Dossier de commande.

58. Arch. nat., F 21 2088. Dossier de commande, lettre au directeur des Beaux-Arts du 16 mai 1887.

59. Arch. nat., F 21 2124. Dossier de commande.

60. Daniel Fabre et Noël Barbe, "Pasteur en héros. L'institution d'un patrimoine scientifique?", séance introductive de l'atelier. Voir : http://www.lahic.cnrs.fr/spip.php ?article380

\section{RÉSUMÉS}

La recherche publiée dans la présente livraison de In Situ s'inscrivait à l'origine dans un atelier du Lahic consacré à la construction de la figure de Pasteur comme héros scientifique. Au sein de ce programme, on s'est plus particulièrement attaché à l'étude de la représentation de Pasteur dans le monde de l'enseignement supérieur parisien. Issu de ce monde avant que d'en être devenu l'une des figures les plus illustres, Louis Pasteur fait l'objet de nombreuses figurations dans les facultés et les grandes écoles parisiennes. Présent dans une dizaine d'établissements - ce qui est rarissime, la plupart des personnages représentés ne l'étant que dans une voire deux institutions - Pasteur marque de son visage aisément reconnaissable les couloirs de la Sorbonne comme le jardin de l'École normale supérieure. Parcourir la Montagne Sainte-Geneviève à la 
recherche de son image renseigne aussi bien sur la figuration de cet éminent savant en héros que sur les relations entretenues, à travers lui, par les différents établissements entre eux : tout entier accaparé par les normaliens et les scientifiques, Pasteur est singulièrement absent chez les médecins, à quelques dizaines de mètres de la Sorbonne...

The research published in this present issue of the electronic review In Situ results from a series of papers discussed in a workshop of the LAHIC laboratory devoted to the question of how Pasteur came to be seen as a scientific hero. Within this programme we were particularly interested in looking at how Pasteur was represented in the world of higher learning in Paris. Pasteur was very much a part of this world before becoming one of its most illustrious representatives and, as such, was the object of numerous representations in the faculties and 'grandes écoles' in Paris. Exceptionally, he was associated with about ten of these institutions; most of the outstanding scientific figures of his time were connected to only one or at most two such institutions. So Pasteur's features were familiar both in the corridors of the Sorbonne and in the gardens of the Ecole normale supérieure. Exploring the Montagne Sainte-Geneviève in search of Pasteur's image gives us information not only on how this eminent man of science was represented but also on the relations between the different institutions he frequented. Largely monopolised by 'normaliens' and by men of science, Pasteur is surprisingly absent from the world of medicine, even though the medical faculty is but a stone's throw from the Sorbonne...

\section{INDEX}

Mots-clés : Louis Pasteur, Sorbonne, École normale supérieure, Louis-Édouard Fournier, patrimoine scientifique

Keywords : scientific heritage

\section{AUTEUR}

\section{CHRISTIAN HOTTIN}

Chef de la mission ethnologie, direction de l'Architecture et du Patrimoine, ministère de la Culture et de la Communication. christian.hottin@culture.gouv.fr 